Article

\title{
Andean Blueberry of the Genus Disterigma: A High-Resolution Mass Spectrometric Approach for the Comprehensive Characterization of Phenolic Compounds
}

\author{
Sara Elsa Aita ${ }^{1}$, Anna Laura Capriotti ${ }^{1}$, Chiara Cavaliere ${ }^{1} \mathbb{D}$, Andrea Cerrato ${ }^{1, *} \mathbb{C}$, Benedetta Giannelli Moneta ${ }^{1}$, \\ Carmela Maria Montone ${ }^{1} \mathbb{D}$, Susy Piovesana ${ }^{1}$ and Aldo Laganà ${ }^{1,2} \mathbb{D}$ \\ 1 Department of Chemistry, Sapienza University of Rome, Piazzale Aldo Moro 5, 00185 Rome, Italy; \\ saraelsa.aita@uniroma1.it (S.E.A.); annalaura.capriotti@uniroma1.it (A.L.C.); \\ chiara.cavaliere@uniroma1.it (C.C.); benedetta.giannellimoneta@uniroma1.it (B.G.M.); \\ carmelamaria.montone@uniroma1.it (C.M.M.); susy.piovesana@uniroma1.it (S.P.); \\ aldo.lagana@uniroma1.it (A.L.) \\ 2 CNR NANOTEC, Campus Ecotekne, University of Salento, Via Monteroni, 73100 Lecce, Italy \\ * Correspondence: andrea.cerrato@uniroma1.it
}

Citation: Aita, S.E.; Capriotti, A.L.; Cavaliere, C.; Cerrato, A.; Giannelli Moneta, B.; Montone, C.M.;

Piovesana, S.; Laganà, A. Andean Blueberry of the Genus Disterigma: A High-Resolution Mass Spectrometric Approach for the Comprehensive Characterization of Phenolic Compounds. Separations 2021, 8, 58 https://doi.org/10.3390/ separations 8050058

\section{Academic Editors: Eduardo}

Sommella, Slawomir Neffe and Zygfryd Witkiewicz

Received: 31 March 2021

Accepted: 29 April 2021

Published: 2 May 2021

Publisher's Note: MDPI stays neutral with regard to jurisdictional claims in published maps and institutional affiliations.

Copyright: (c) 2021 by the authors. Licensee MDPI, Basel, Switzerland. This article is an open access article distributed under the terms and conditions of the Creative Commons Attribution (CC BY) license (https:/ / creativecommons.org/licenses/by/ $4.0 /)$.

\begin{abstract}
Wild neotropical blueberries, endemic of Central and South American areas, are promising yet still undisclosed sources of bioactive compounds. Most research studies have addressed wild and cultivated blueberries from Europe and North America, despite the extremely wide variety of wild neotropical species. In the present paper, for the first time, the phenolic composition of Disterigma alaternoides was investigated through ultra-high-performance liquid chromatography coupled to highresolution mass-spectrometric analysis followed by accurate data analysis and compound validation with a dedicated structure-based workflow. D. alaternoides, which belongs to a closely related genus to that of the common blueberry, grows exclusively in the Andean regions over 2000 above sea level. Thanks to the dedicated analytical platform, 249 phenolic compounds were tentatively identified, including several anthocyanins, flavonoids, phenolic acids, and proanthocyanidins. Thenature and heterogeneity of identified phenolic compounds demonstrate once more the need for a more profound knowledge of such still uncharted matrices.
\end{abstract}

Keywords: polyphenols; neotropical berries; Disterigma alaternoides; anthocyanins; Compound Discoverer

\section{Introduction}

The vast majority of the research activity on the berries of the plant family Ericaceae has addressed temperate species of Vaccinium [1-3], which is only 1 of the 32 genera of the tribe Vacciniae of the family Ericaceae. Among these species, the most known are blueberry (Vaccinium Corymbosum) [4], bilberry (Vaccinium myrtillus) [5], cranberry (Vaccinium macrocarpon) [6], and lingonberry (Vaccinium vitis-idaea) [7]. Nevertheless, more than 600 species of berry-producing Ericaceae are native to the Neotropical realm, including South America, Central America, and the Caribbean islands [8]. Several neotropical blueberries in the Andean region of South America are widely consumed raw or in different preparations [9].

Berries of the genus Vaccinium have been raising interest for their extremely high content in flavonoids, anthocyanins, phenolic acids, and tannins, which have been demonstrated to exert a wide range of biological activities [10,11]. In a recent paper by Rutledge et al. [12], blueberry phenolics were associated with a cognitive enhancement in healthy elder adults. Likewise, Stull et al. [13] reported that consumption of the whole blueberry reduces the blood glucose level in vivo. For these reasons, blueberries are often referred to as "super-fruits". At present, the composition of blueberries from North American and European regions has been widely investigated $[4,14,15]$. In a recent study by Ancillotti et al. [2], the polyphenol composition of cultivated V. corymbosum and wild V. myrtillus and $V$. uliginosum were evaluated by liquid chromatography coupled to high-resolution mass 
spectrometry (HRMS). More than 200 compounds were tentatively identified in the hydroalcoholic extracts, comprising mainly anthocyanins, flavonols, and proanthocyanidins, with the wild berries presenting generally higher concentrations. As well as the common species of blueberries, other species native to South America have been the object of several studies. V. floribundum, a woody perennial shrub that is endemic in the Andean region and grows between 1600 and $4500 \mathrm{~m}$ above sea level (masl) [16], has been extensively studied for its potential beneficial effects and its vast consumption by the local population [17-20]. Despite the growing interest in the bioactive compounds in berries from South America, there is still a lack of knowledge in the phenol composition of berries belonging to other genera of the Ericaceae family. In the present paper, for the first time, the phenolic compound composition of Disterigma alaternoides was determined by ultra-high-performance liquid chromatography (UHPLC) coupled to HRMS. The genus Disterigma has more than 35 species of small shrubs, distributed from southern Mexico to Bolivia, generally above 2000 masl [21]. The paper aims to widen the knowledge on often neglected species that could possess peculiar characteristics since they grow in the unique Andean ecosystems.

\section{Materials and Methods}

\subsection{Samples, Chemicals, and Reagents}

D. alaternoides fruit samples were obtained by the National Agrarian University La Molina (Lima, Perú). Their taxonomy was certified by the Herbario San Marcos (National University of San Marcos, Lima, Perú). Berries were mashed, freeze-dried by a Heto PowerDry LL1500 (Thermo Fisher), finely ground in a mortar, and stored at $-20^{\circ} \mathrm{C}$ until use. Optima ${ }^{\circledR}$ LC-MS grade water, methanol $(\mathrm{MeOH})$, and acetonitrile $(\mathrm{ACN})$ were purchased from Thermo Fisher Scientific (Waltham, MA, USA). Acetone, acetic acid, formic acid, and sodium hypochlorite were purchased from Merck (Kenilworth, NJ, USA).

\subsection{Phenolic Compound Extraction}

Freeze-dried berries were extracted as previously reported with slight modifications [22]. Briefly, $0.2 \mathrm{~g}$ of freeze-dried berry samples were extracted with $10 \mathrm{~mL}$ $\mathrm{CH}_{3} \mathrm{COCH}_{3} / \mathrm{H}_{2} \mathrm{O} / \mathrm{CH}_{3} \mathrm{COOH}(70: 29.5: 0.5, v / v / v)$. The extract was sonicated for $15 \mathrm{~min}$ in an ice bath and then centrifuged for $10 \mathrm{~min}$ at $2000 \times \mathrm{g}$. The supernatant was collected, and the procedure was repeated once. The supernatants were mixed and concentrated to $4.5 \mathrm{~mL}$ using a Speed-Vac SC 250 Express (Thermo 164 Avant, Holbrook, NY, USA). Then, $500 \mu \mathrm{L}$ of $\mathrm{MeOH}$ was added to the sample, and the final extract solution $\left(\mathrm{H}_{2} \mathrm{O} / \mathrm{MeOH}\right.$, 90:10 $v / v$ ) was filtered through a 13-mm Acrodisc Syringe filter with a $0.2 \mu \mathrm{m}$ GH Polypro membrane (Pall, Ann Arbor, MI, USA). Finally, the extract was aliquoted and stored at $-20^{\circ} \mathrm{C}$ for further analysis.

\subsection{UHPLC-HRMS Analysis}

Phenolic compound chromatographic separation was carried out by a Vanquish binary pump H (Thermo Fisher Scientific, Bremen, Germany), equipped with a thermostated autosampler and column compartment, on a Kinetex core-shell C18 column (100 $\mathrm{mm} \times 2.1 \mathrm{~mm}$ i.d.) with a particle size of $2.6 \mu \mathrm{m}$ (Phenomenex, Torrance, CA, USA) at $40{ }^{\circ} \mathrm{C}$ and with a flow-rate of $600 \mu \mathrm{L} \mathrm{min}{ }^{-1}$. The injection volume was $10 \mu \mathrm{L}$. The mobile phases consisted of $\mathrm{H}_{2} \mathrm{O} / \mathrm{HCOOH}$ (99.9:0.1, v/v; phase A) and ACN/HCOOH (99.9:0.1, $v / v$; phase B). The elution gradient was optimized in a previous study [22]. The chromatographic system was coupled to a $Q$ Exactive hybrid quadrupole-Orbitrap mass spectrometer (Thermo Fisher Scientific) with a heated ESI source. The ESI source parameters were set as reported in our previous work [23]. The detection was conducted in TOP 5 data-dependent acquisition (DDA) mode for low- and high-molecular-weight phenolic compounds. An exclusion list containing the most intense ions detected in the blank sample, consisting of $\mathrm{H}_{2} \mathrm{O} / \mathrm{MeOH}(90: 10, v / v)$, was added to the mass-spectrometric method. For low-molecularweight phenolic compound analysis (flavonoids, anthocyanins, and phenolic acids) and high-molecular-weight polyphenol analysis (tannins), MS data were acquired in the range 
150-1000 m/z and 300-2000 m/z, respectively, with a resolution (full width at half maximum, FWHM, at $m / z 200$ ) of 70,000. In full scan mode, the automatic gain control (AGC) target value was 200,000 and the maximum ion injection time was $100 \mathrm{~ms}$. The isolation window width was $2 \mathrm{~m} / z$. MS/MS fragmentation was performed with a resolution (FWHM, at $m / z 200$ ) of 35,000 with AGC target value set at 100,000 and dynamic exclusion set to $3 \mathrm{~s}$. Fragmentation was achieved in the higher-collision dissociation (HCD) cell at three values of normalized collision energy (NCE), namely, 20-50-80 NCE in the positive ion mode and 20-40-60 NCE in the negative ion mode, based on the results of a previous study [24]. All samples were run in triplicate.

\subsection{Phenolic Compound Identification}

Raw data obtained from three consecutive injections and the blank sample were processed by Compound Discoverer 3.1 (Thermo Fisher Scientific) using a customized method specifically dedicated to phenolic compound analysis [24,25]. Customized databases were generated by combining free phenolic compounds (aglycones) with a series of sugars, aliphatic, and aromatic acids, and complete IDs, accurate masses, and molecular formulas were implemented in the mass list feature for the automatic matching of extracted $m / z$ ratios (45,567 combinations). Moreover, detailed HCD fragmentation spectra for flavonoids and phenolic acids were implemented in the compound class scoring section for automatic MS/MS spectra matching. The parameters for the predict composition tool were adapted to phenolic compounds. Extracted $\mathrm{m} / \mathrm{z}$ from the raw chromatograms were grouped, aligned, and filtered to remove background compounds found in the blank sample, $m / z$ values not associated with compounds present in the databases, and the features lacking MS/MS spectra. Filtered compounds were manually validated by matching fragmentation spectra to those of available standards or spectra reported in the literature. When data were lacking, phenolic compounds were tentatively identified according to the characteristic fragmentation spectra. The identification data for the tentatively identified compounds are discussed in the following sections and summarized in Tables 1-4 and Tables S1-S4 with the related confidence level according to Schymanski et al. [26].

Table 1. Retention times (Rt, min), proposed formulas, experimental $m / z$, accuracy $(\Delta, \mathrm{ppm})$, main diagnostic experimental product ions, and confidence level of the identification (c. 1.) of the 18 tentatively identified anthocyanins in Disterigma alaternoides extract in $\mathrm{ESI}(+)$.

\begin{tabular}{|c|c|c|c|c|c|c|c|}
\hline Id & Name & Rt (min) & $\begin{array}{l}\text { Proposed } \\
\text { Formula }\end{array}$ & $\begin{array}{l}\text { Experimental } \\
\mathrm{m} / \mathrm{z}\end{array}$ & $\begin{array}{l}\Delta \text { mass } \\
(\mathrm{ppm})\end{array}$ & $\begin{array}{l}\text { Diagnostic Product Ions } \\
\qquad(\mathrm{m} / \mathrm{z})\end{array}$ & c. 1. \\
\hline 1 & Cyanidin O-hexoside (I) & 1.63 & $\mathrm{C}_{21} \mathrm{H}_{21} \mathrm{O}_{11}{ }^{+}$ & 449.1088 & 1.5 & $\begin{array}{c}287.0553 ; 231.0656 ; 213.0550 \\
149.0237 ; 137.0235\end{array}$ & 2 \\
\hline 2 & Delphinidin O-hexoside (I) & 2.79 & $\mathrm{C}_{21} \mathrm{H}_{21} \mathrm{O}_{12}{ }^{+}$ & 465.1038 & 2.1 & $\begin{array}{c}303.0502 ; 247.0605 ; 229.0499 \\
153.0185 ; 149.0237\end{array}$ & 2 \\
\hline 3 & Delphinidin O-hexoside (II) & 3.77 & $\mathrm{C}_{21} \mathrm{H}_{21} \mathrm{O}_{12}{ }^{+}$ & 465.1034 & 1.4 & $\begin{array}{c}303.0502 ; 247.0605 ; 229.0499 \\
153.0185 ; 149.0237\end{array}$ & 2 \\
\hline 4 & Delphinidin O-pentoside & 4.98 & $\mathrm{C}_{20} \mathrm{H}_{19} \mathrm{O}_{11}^{+}$ & 435.0920 & -0.5 & $\begin{array}{c}\text { 303.0502; } 247.0605 ; 229.0499 \\
153.0185 ; 149.0237\end{array}$ & 2 \\
\hline 5 & Cyanidin O-hexoside (II) & 5.07 & $\mathrm{C}_{21} \mathrm{H}_{21} \mathrm{O}_{11}{ }^{+}$ & 449.1088 & 2.1 & $\begin{array}{c}287.0553 ; 231.0656 ; 213.0550 \\
149.0237137 .0235\end{array}$ & 2 \\
\hline 6 & Delphinidin O-hexoside (III) & 5.95 & $\mathrm{C}_{21} \mathrm{H}_{21} \mathrm{O}_{12}{ }^{+}$ & 465.1038 & 2.1 & $\begin{array}{c}\text { 303.0502; } 247.0605 ; 229.0499 \\
153.0185 ; 149.0237\end{array}$ & 2 \\
\hline 7 & Pelargonidin O-hexoside & 6.25 & $\mathrm{C}_{21} \mathrm{H}_{21} \mathrm{O}_{10}{ }^{+}$ & 433.1127 & -0.4 & $\begin{array}{c}\text { 271.0608; 215.0707; } 197.0601 \\
\text { 149.0237; } 121.0285\end{array}$ & 2 \\
\hline 8 & Cyanidin O-deoxyhexoside & 6.54 & $\mathrm{C}_{21} \mathrm{H}_{21} \mathrm{O}_{10}{ }^{+}$ & 433.1126 & 1.9 & $\begin{array}{c}287.0553 ; 231.0656 ; 213.0550 \\
149.0237 ; 137.0235\end{array}$ & 2 \\
\hline 9 & Cyanidin O-pentoside (I) & 6.71 & $\mathrm{C}_{20} \mathrm{H}_{19} \mathrm{O}_{10}{ }^{+}$ & 419.0976 & 0.8 & $\begin{array}{c}287.0553 ; 231.0656 ; 213.0550 \\
149.0237 ; 137.0235\end{array}$ & 2 \\
\hline 10 & Pelargonidin O-pentoside & 7.66 & $\mathrm{C}_{20} \mathrm{H}_{19} \mathrm{O}_{9}^{+}$ & 403.1030 & 1.6 & $\begin{array}{c}271.0608 ; 215.0707 ; 197.0601 \\
149.0237 ; 121.0285\end{array}$ & 2 \\
\hline 11 & Cyanidin O-hexoside (III) & 7.98 & $\mathrm{C}_{21} \mathrm{H}_{21} \mathrm{O}_{11}{ }^{+}$ & 449.1088 & 2.2 & $\begin{array}{c}287.0553 ; 231.0656 ; 213.0550 \\
149.0237 ; 137.0235\end{array}$ & 2 \\
\hline 12 & Peonidin 3-O-glucoside & 8.34 & $\mathrm{C}_{22} \mathrm{H}_{23} \mathrm{O}_{11}^{+}$ & 463.1247 & 2.7 & $\begin{array}{c}301.0707 ; 286.0472 ; 149.0237 \\
121.0285\end{array}$ & 1 \\
\hline
\end{tabular}


Table 1. Cont.

\begin{tabular}{|c|c|c|c|c|c|c|c|}
\hline Id & Name & Rt (min) & $\begin{array}{l}\text { Proposed } \\
\text { Formula }\end{array}$ & $\begin{array}{l}\text { Experimental } \\
\mathrm{m} / \mathrm{z}\end{array}$ & $\begin{array}{l}\Delta \text { mass } \\
(\mathrm{ppm})\end{array}$ & $\begin{array}{l}\text { Diagnostic Product Ions } \\
(\mathrm{m} / \mathrm{z})\end{array}$ & c. 1 . \\
\hline 13 & Peonidin O-pentoside & 8.75 & $\mathrm{C}_{21} \mathrm{H}_{21} \mathrm{O}_{10}{ }^{+}$ & 433.1136 & 1.5 & $\begin{array}{c}301.0707 ; \text { 2 } 286.0472 ; 149.0237 \\
121.0285\end{array}$ & 2 \\
\hline 14 & Cyanidin O-hexoside (IV) & 8.88 & $\mathrm{C}_{21} \mathrm{H}_{21} \mathrm{O}_{11}{ }^{+}$ & 449.1088 & 2.1 & $\begin{array}{c}\text { 287.0553; 231.0656; } 213.0550 \\
\text { 149.0237; } 137.0235\end{array}$ & 2 \\
\hline 15 & Delphinidin O-dihexoside & 9.40 & $\mathrm{C}_{27} \mathrm{H}_{31} \mathrm{O}_{17}+$ & 627.1570 & 2.2 & $\begin{array}{c}\text { 303.0502; } 247.0605 ; 229.0499 \\
153.0185 ; 149.0237\end{array}$ & 2 \\
\hline 16 & Cyanidin O-pentoside (II) & 9.51 & $\mathrm{C}_{20} \mathrm{H}_{19} \mathrm{O}_{10}{ }^{+}$ & 419.0976 & 0.8 & $\begin{array}{c}287.0553 ; 231.0656 ; 213.0550 \\
149.0237 ; 137.0235\end{array}$ & 2 \\
\hline 17 & Cyanidin & 10.23 & $\mathrm{C}_{15} \mathrm{H}_{11} \mathrm{O}_{6}^{+}$ & 287.0541 & -3.2 & $\begin{array}{c}287.0553 ; 231.0656 ; 213.0550 \\
149.0237 ; 137.0235\end{array}$ & 1 \\
\hline 18 & Cyanidin isomer & 12.06 & $\mathrm{C}_{15} \mathrm{H}_{11} \mathrm{O}_{6}^{+}$ & 287.0541 & -3.2 & $\begin{array}{l}\text { 287.0553; } 231.0656 ; 213.0550 \\
149.0237 ; 147.0445 ; 137.0235\end{array}$ & 2 \\
\hline
\end{tabular}

Table 2. Retention times (Rt, min), proposed formulas, experimental $m / z$, accuracy $(\Delta, \mathrm{ppm})$, main diagnostic experimental product ions, and confidence level of the identification (c. 1.) of the 87 tentatively identified flavonoids in Disterigma alaternoides extract in $\operatorname{ESI}(-)$.

\begin{tabular}{|c|c|c|c|c|c|c|c|}
\hline Id & Name & Rt (min) & $\begin{array}{l}\text { Proposed } \\
\text { Formula }\end{array}$ & $\begin{array}{l}\text { Experimental } \\
\mathrm{m} / \mathrm{z}\end{array}$ & $\Delta(\mathrm{ppm})$ & $\begin{array}{l}\text { Diagnostic Product Ions } \\
(\mathrm{m} / \mathrm{z})\end{array}$ & c. 1 . \\
\hline 19 & (Epi)catechin $O$-hexoside & 3.47 & $\mathrm{C}_{21} \mathrm{H}_{24} \mathrm{O}_{11}$ & 451.1253 & 1.6 & $\begin{array}{c}289.0719 ; 245.0817 ; 137.0245 \\
125.0244\end{array}$ & 2 \\
\hline 20 & Epicatechin & 6.30 & $\mathrm{C}_{15} \mathrm{H}_{14} \mathrm{O}_{6}$ & 289.0724 & 1.8 & $\begin{array}{c}289.0719 ; 245.0817 ; 137.0245 \\
125.0244 ;\end{array}$ & 1 \\
\hline 21 & Taxifolin isomer & 7.05 & $\mathrm{C}_{15} \mathrm{H}_{12} \mathrm{O}_{7}$ & 303.0515 & 1.7 & $\begin{array}{c}259.0613 ; 193.0142 ; 167.0350 \\
\text { 165.0193; } 137.0245\end{array}$ & 2 \\
\hline 22 & Quercetin $O$-dihexoside & 7.05 & $\mathrm{C}_{27} \mathrm{H}_{30} \mathrm{O}_{17}$ & 625.1430 & 3.1 & $\begin{array}{c}301.0354 ; 178.9985 ; 151.0036 \\
121.0295\end{array}$ & 2 \\
\hline 23 & Aromadendrin $O$-hexoside & 7.25 & $\mathrm{C}_{21} \mathrm{H}_{22} \mathrm{O}_{11}$ & 449.1100 & 2.4 & $\begin{array}{c}287.0561 ; 151.0036 ; 125.0244 \\
107.0139\end{array}$ & 2 \\
\hline 24 & Taxifolin $O$-hexoside & 7.65 & $\mathrm{C}_{21} \mathrm{H}_{22} \mathrm{O}_{12}$ & 465.1057 & 4.0 & $\begin{array}{c}\text { 303.0512; 177.0194; 151.0036; } \\
\text { 125.0244 }\end{array}$ & 2 \\
\hline 25 & Eriodictyol O-pentoside & 8.21 & $\mathrm{C}_{20} \mathrm{H}_{20} \mathrm{O}_{10}$ & 419.0995 & 2.7 & $\begin{array}{c}287.0561 ; 177.0194 ; 151.0036 \\
135.0452\end{array}$ & 2 \\
\hline 26 & $\begin{array}{l}\text { Quercetin O-hexoside } \\
\text { O-deoxyhexoside }\end{array}$ & 8.70 & $\mathrm{C}_{27} \mathrm{H}_{30} \mathrm{O}_{16}$ & 609.1475 & 2.3 & $\begin{array}{l}\text { 463.0859; 447.0949; 301.0354; } \\
\text { 178.9985; 151.0036; } 121.0295\end{array}$ & 2 \\
\hline 27 & Taxifolin & 9.26 & $\mathrm{C}_{15} \mathrm{H}_{12} \mathrm{O}_{7}$ & 303.0520 & 3.3 & $\begin{array}{c}\text { 303.0512; 241.0407; } 177.0194 \\
\text { 151.0036; } 125.0244\end{array}$ & 1 \\
\hline 28 & Quercetin O-dihexoside & 9.38 & $\mathrm{C}_{27} \mathrm{H}_{30} \mathrm{O}_{17}$ & 625.1430 & 3.2 & $\begin{array}{c}\text { 463.0880; 301.0354; 178.9985; } \\
\text { 151.0036; } 121.0295\end{array}$ & 2 \\
\hline 29 & Myricetin 3-O-hexoside & 9.55 & $\mathrm{C}_{21} \mathrm{H}_{20} \mathrm{O}_{13}$ & 479.0854 & 2.7 & $\begin{array}{c}\text { 317.0303; 316.0242; } 178.9985 \\
\text { 151.0036; } 137.0244\end{array}$ & 2 \\
\hline 30 & Myricetin $O$-pentoside & 9.58 & $\mathrm{C}_{20} \mathrm{H}_{18} \mathrm{O}_{12}$ & 449.0738 & 2.7 & $\begin{array}{c}\text { 317.0303; 178.9985; 151.0036; } \\
137.0244\end{array}$ & 2 \\
\hline 31 & Myricetin 3-O-hexoside & 9.86 & $\mathrm{C}_{21} \mathrm{H}_{20} \mathrm{O}_{13}$ & 479.0844 & 2.6 & $\begin{array}{c}\text { 317.0303; 316.0242; } 178.9985 \\
151.0036 ; 137.0244\end{array}$ & 2 \\
\hline 32 & Isorhamnetin O-glucuronide & 9.96 & $\mathrm{C}_{22} \mathrm{H}_{20} \mathrm{O}_{13}$ & 491.0844 & 2.7 & $\begin{array}{c}315.0512 ; 300.0275 ; 255.0296 \\
151.0036\end{array}$ & 2 \\
\hline 33 & Naringenin $O$-hexoside & 10.03 & $\mathrm{C}_{21} \mathrm{H}_{22} \mathrm{O}_{10}$ & 433.1150 & 2.2 & $\begin{array}{c}271.0613 ; 227.0713 ; 177.0194 \\
151.0036 ; 119.0503\end{array}$ & 2 \\
\hline 34 & $\begin{array}{l}\text { Myricetin O-hexoside } \\
\text { O-pentoside }\end{array}$ & 10.04 & $\mathrm{C}_{26} \mathrm{H}_{28} \mathrm{O}_{17}$ & 611.1266 & 2.0 & $\begin{array}{c}\text { 479.0833; 449.0729; 317.0303; } \\
316.0242 ; 178.9985 ; 151.0036 \\
137.0244\end{array}$ & 2 \\
\hline 35 & Myricetin $O$-pentoside & 10.07 & $\mathrm{C}_{20} \mathrm{H}_{18} \mathrm{O}_{12}$ & 449.0735 & 2.0 & $\begin{array}{c}\text { 317.0303; 178.9985; 151.0036; } \\
\text { 137.0244 }\end{array}$ & 2 \\
\hline 36 & Quercetin O-dihexoside & 10.08 & $\mathrm{C}_{27} \mathrm{H}_{30} \mathrm{O}_{17}$ & 625.1426 & 2.6 & $\begin{array}{l}463.0880 ; 301.0354 ; 300.0278 \\
178.9985 ; 151.0036 ; 121.0295\end{array}$ & 2 \\
\hline 37 & Myricetin $O$-pentoside & 10.16 & $\mathrm{C}_{20} \mathrm{H}_{18} \mathrm{O}_{12}$ & 449.0736 & 2.2 & $\begin{array}{c}\text { 317.0303; } 178.9985 ; 151.0036 \\
137.0244\end{array}$ & 2 \\
\hline 38 & $\begin{array}{l}\text { Quercetin O-hexoside } \\
\text { O-pentoside }\end{array}$ & 10.33 & $\mathrm{C}_{26} \mathrm{H}_{28} \mathrm{O}_{16}$ & 595.1314 & 1.5 & $\begin{array}{l}\text { 463.0901; 433.0777; 301.0354; } \\
\text { 178.9985; 151.0036; } 121.0295\end{array}$ & 2 \\
\hline 39 & Taxifolin isomer & 10.36 & $\mathrm{C}_{15} \mathrm{H}_{12} \mathrm{O}_{7}$ & 303.0520 & 3.2 & $\begin{array}{c}285.0407 ; 241.0407 ; 177.0194 \\
\text { 151.0036; } 125.0244\end{array}$ & 2 \\
\hline 40 & $\begin{array}{l}\text { Quercetin } \\
\text { O-hexosylpentoside }\end{array}$ & 10.62 & $\mathrm{C}_{26} \mathrm{H}_{28} \mathrm{O}_{16}$ & 595.1313 & 1.4 & $\begin{array}{c}\text { 301.0354; 300.0278; } 178.9985 \\
151.0036 ; 121.0295\end{array}$ & 2 \\
\hline
\end{tabular}


Table 2. Cont.

\begin{tabular}{|c|c|c|c|c|c|c|c|}
\hline Id & Name & Rt (min) & $\begin{array}{l}\text { Proposed } \\
\text { Formula }\end{array}$ & $\begin{array}{l}\text { Experimental } \\
\mathrm{m} / \mathrm{z}\end{array}$ & $\Delta(\mathrm{ppm})$ & $\begin{array}{l}\text { Diagnostic Product Ions } \\
\qquad(\mathrm{m} / \mathrm{z})\end{array}$ & c. 1 . \\
\hline 41 & Myricetin isomer & 10.63 & $\mathrm{C}_{15} \mathrm{H}_{10} \mathrm{O}_{8}$ & 317.0308 & 1.7 & $\begin{array}{c}\text { 317.0303; } 178.9985 ; 151.0036 \\
137.0244\end{array}$ & 2 \\
\hline 42 & Naringenin O-hexoside & 10.81 & $\mathrm{C}_{21} \mathrm{H}_{22} \mathrm{O}_{10}$ & 433.1144 & 0.9 & $\begin{array}{c}\text { 271.0613; 227.0713; } 177.0194 \\
\text { 151.0036; } 119.0503\end{array}$ & 2 \\
\hline 43 & Eriodictyol O-hexoside & 10.84 & $\mathrm{C}_{21} \mathrm{H}_{22} \mathrm{O}_{11}$ & 449.1101 & 2.7 & $\begin{array}{c}287.0561 ; 177.0194 ; 151.0036 \\
135.0452\end{array}$ & 2 \\
\hline 44 & Shiikimoyl Kaempferol & 11.10 & $\mathrm{C}_{22} \mathrm{H}_{18} \mathrm{O}_{10}$ & 441.0838 & 2.5 & $\begin{array}{c}285.0407 ; 243.0296 ; 241.0502 \\
151.0036 ; 133.0295\end{array}$ & 2 \\
\hline 45 & Myricetin 3-O-pentoside & 11.11 & $\mathrm{C}_{20} \mathrm{H}_{18} \mathrm{O}_{12}$ & 449.0735 & 2.0 & $\begin{array}{c}\text { 317.0303; 316.0242; } 178.9985 \\
151.0036 ; 137.0244\end{array}$ & 2 \\
\hline 46 & $\begin{array}{l}\text { Quercetin } O \text {-hexoside } \\
O \text {-pentoside }\end{array}$ & 11.18 & $\mathrm{C}_{26} \mathrm{H}_{28} \mathrm{O}_{16}$ & 595.1310 & 1.0 & $\begin{array}{l}\text { 463.0901; 433.0777; 301.0354; } \\
\text { 178.9985; 151.0036; } 121.0295\end{array}$ & 2 \\
\hline 47 & Myricetin $O$-pentoside & 11.21 & $\mathrm{C}_{20} \mathrm{H}_{18} \mathrm{O}_{12}$ & 449.0724 & -0.4 & $\begin{array}{c}\text { 317.0303; } 178.9985 ; 151.0036 \\
137.0244\end{array}$ & 2 \\
\hline 48 & Myricetin 3-O-deoxyhexoside & 11.25 & $\mathrm{C}_{21} \mathrm{H}_{20} \mathrm{O}_{12}$ & 463.0895 & 2.8 & $\begin{array}{c}\text { 317.0303; 316.0242; 178.9985; } \\
\text { 151.0036; 137.0244; }\end{array}$ & 2 \\
\hline 49 & $\begin{array}{l}\text { Quercetin } O \text {-hexoside } \\
\text { O-pentoside }\end{array}$ & 11.27 & $\mathrm{C}_{26} \mathrm{H}_{28} \mathrm{O}_{16}$ & 595.1315 & 1.8 & $\begin{array}{l}\text { 463.0901; 433.0777; 301.0354; } \\
\text { 178.9985; 151.0036; } 121.0295\end{array}$ & 2 \\
\hline 50 & $\begin{array}{l}\text { Quercetin } O \text {-hexoside } \\
O \text {-pentoside }\end{array}$ & 11.55 & $\mathrm{C}_{26} \mathrm{H}_{28} \mathrm{O}_{16}$ & 595.1321 & 2.7 & $\begin{array}{l}\text { 463.0901; 433.0777; 301.0354; } \\
\text { 178.9985; 151.0036; } 121.0295\end{array}$ & 2 \\
\hline 51 & Quercetin 3-O-galactoside & 11.58 & $\mathrm{C}_{21} \mathrm{H}_{20} \mathrm{O}_{12}$ & 463.0883 & 0.2 & $\begin{array}{c}\text { 301.0354; 300.0278; } 178.9985 \\
\text { 151.0036; } 121.0295\end{array}$ & 2 \\
\hline 52 & $\begin{array}{l}\text { Quercetin O-hexoside } \\
\text { O-pentoside }\end{array}$ & 11.69 & $\mathrm{C}_{26} \mathrm{H}_{28} \mathrm{O}_{16}$ & 595.1319 & 2.3 & $\begin{array}{l}\text { 463.0901; 433.0777; 301.0354; } \\
\text { 178.9985; 151.0036; } 121.0295\end{array}$ & 2 \\
\hline 53 & $\begin{array}{l}\text { Quercetin } O \text {-hexoside } \\
\text { O-pentoside }\end{array}$ & 11.84 & $\mathrm{C}_{26} \mathrm{H}_{28} \mathrm{O}_{16}$ & 595.1321 & 2.7 & $\begin{array}{l}\text { 463.0901; 433.0777; 301.0354; } \\
\text { 178.9985; 151.0036; 121.0295 }\end{array}$ & 2 \\
\hline 54 & Rutin & 11.90 & $\mathrm{C}_{27} \mathrm{H}_{30} \mathrm{O}_{16}$ & 609.1482 & 3.5 & $\begin{array}{c}463.0859 ; 301.0354 ; 178.9985 \\
151.0036 ; 121.0295\end{array}$ & 1 \\
\hline 55 & Quercetin 3-O-glucoside & 12.09 & $\mathrm{C}_{21} \mathrm{H}_{20} \mathrm{O}_{12}$ & 463.0884 & 0.4 & $\begin{array}{c}\text { 301.0354; 300.0278; 178.9985; } \\
\text { 151.0036; } 121.0295\end{array}$ & 1 \\
\hline 56 & Aromadendrin & 12.15 & $\mathrm{C}_{15} \mathrm{H}_{12} \mathrm{O}_{6}$ & 287.0570 & 3.1 & $\begin{array}{c}287.0561 ; 269.0456 ; 177.0194 \\
151.0036 ; 125.0244\end{array}$ & 2 \\
\hline 57 & Quercetin O-dipentoside & 12.26 & $\mathrm{C}_{25} \mathrm{H}_{26} \mathrm{O}_{15}$ & 565.1212 & 2.3 & $\begin{array}{c}\text { 433.0776; 301.0354; } 178.9985 \\
\text { 151.0036; } 121.0295\end{array}$ & 2 \\
\hline 58 & $\begin{array}{l}\text { Quercetin } O \text {-hexoside } \\
\text { O-pentoside }\end{array}$ & 12.30 & $\mathrm{C}_{26} \mathrm{H}_{28} \mathrm{O}_{16}$ & 595.1318 & 2.3 & $\begin{array}{l}\text { 463.0901; 433.0777; 301.0354; } \\
178.9985 ; 151.0036 ; 121.0295\end{array}$ & 2 \\
\hline 59 & $\begin{array}{l}\text { Quercetin } O \text {-hexoside } \\
O \text {-pentoside }\end{array}$ & 12.37 & $\mathrm{C}_{26} \mathrm{H}_{28} \mathrm{O}_{16}$ & 595.1317 & 2.0 & $\begin{array}{l}\text { 463.0901; 433.0777; 301.0354; } \\
\text { 178.9985; 151.0036; } 121.0295\end{array}$ & 2 \\
\hline 60 & Kaempferol $O$-hexoside & 12.45 & $\mathrm{C}_{21} \mathrm{H}_{20} \mathrm{O}_{11}$ & 447.0950 & 3.9 & $\begin{array}{c}285.0403 ; 257.0457 ; 229.0504 \\
151.0036\end{array}$ & 2 \\
\hline 61 & Quercetin 3-O-pentoside & 12.62 & $\mathrm{C}_{20} \mathrm{H}_{18} \mathrm{O}_{11}$ & 433.0776 & -0.1 & $\begin{array}{c}\text { 301.0354; 300.0278; } 178.9985 \\
151.0036 ; 121.0295\end{array}$ & 2 \\
\hline 62 & $\begin{array}{l}\text { Quercetin } \\
\text { O-hexosylpentoside }\end{array}$ & 12.87 & $\mathrm{C}_{26} \mathrm{H}_{28} \mathrm{O}_{16}$ & 595.1320 & 2.5 & $\begin{array}{c}\text { 433.0777; 301.0354; } 178.9985 \\
151.0036 ; 121.0295\end{array}$ & 2 \\
\hline 63 & Eriodictyol O-hexoside & 12.91 & $\mathrm{C}_{21} \mathrm{H}_{22} \mathrm{O}_{11}$ & 449.1100 & 2.4 & $\begin{array}{c}287.0561 ; 177.0194 ; 151.0036 \\
135.0452\end{array}$ & 2 \\
\hline 64 & Quercetin $O$-pentoside & 12.99 & $\mathrm{C}_{20} \mathrm{H}_{18} \mathrm{O}_{11}$ & 433.0775 & -0.2 & $\begin{array}{c}\text { 301.0354; } 178.9985 ; 151.0036 \\
121.0295\end{array}$ & 2 \\
\hline 65 & Kaempferol 3-O-hexoside & 13.14 & $\mathrm{C}_{21} \mathrm{H}_{20} \mathrm{O}_{11}$ & 447.0944 & 2.5 & $\begin{array}{c}285.0403 ; 255.0300 ; 227.0351 \\
151.0036\end{array}$ & 2 \\
\hline 66 & Naringenin $O$-hexoside & 13.28 & $\mathrm{C}_{21} \mathrm{H}_{22} \mathrm{O}_{10}$ & 433.1146 & 1.3 & $\begin{array}{c}\text { 271.0613; 227.0713; 177.0194; } \\
\text { 151.0036; } 119.0503\end{array}$ & 2 \\
\hline 67 & Quercetin $O$-dipentoside & 13.36 & $\mathrm{C}_{25} \mathrm{H}_{26} \mathrm{O}_{15}$ & 565.1214 & 2.6 & $\begin{array}{c}\text { 433.0776; 301.0354; } 178.9985 \\
\text { 151.0036; } 121.0295\end{array}$ & 2 \\
\hline 68 & Quercetin O-pentoside & 13.51 & $\mathrm{C}_{20} \mathrm{H}_{18} \mathrm{O}_{11}$ & 433.0775 & -0.3 & $\begin{array}{c}\text { 301.0354; } 178.9985 ; 151.0036 \\
121.0295\end{array}$ & 2 \\
\hline 69 & Quercetin O-pentoside & 13.84 & $\mathrm{C}_{20} \mathrm{H}_{18} \mathrm{O}_{11}$ & 433.0776 & -0.2 & $\begin{array}{c}301.0354 ; 178.9985 ; 151.0036 \\
121.0295\end{array}$ & 2 \\
\hline 70 & Myricetin & 13.89 & $\mathrm{C}_{15} \mathrm{H}_{10} \mathrm{O}_{8}$ & 317.0310 & 2.2 & $\begin{array}{c}\text { 317.0303; } 178.9985 ; 151.0036 \\
\text { 137.0244 }\end{array}$ & 2 \\
\hline 71 & Quercetin 3-O-deoxyhexoside & 14.04 & $\mathrm{C}_{21} \mathrm{H}_{20} \mathrm{O}_{11}$ & 447.0933 & 0.0 & $\begin{array}{c}\text { 301.0354; 300.0278; 178.9985; } \\
\text { 151.0036; } 121.0295\end{array}$ & 2 \\
\hline 72 & Quercetin O-hexoside & 14.18 & $\mathrm{C}_{21} \mathrm{H}_{20} \mathrm{O}_{12}$ & 463.0899 & 3.7 & $\begin{array}{c}301.0354 ; 178.9985 ; 151.0036 \\
121.0295\end{array}$ & 2 \\
\hline 73 & Isorhamnetin 3-O-hexoside & 14.27 & $\mathrm{C}_{22} \mathrm{H}_{22} \mathrm{O}_{12}$ & 477.1052 & 2.8 & $\begin{array}{c}\text { 315.0512; 314.0436; } 300.0275 \\
\text { 271.0244; } 151.0036\end{array}$ & 2 \\
\hline 74 & Kaempferol $O$-pentoside & 14.40 & $\mathrm{C}_{20} \mathrm{H}_{18} \mathrm{O}_{10}$ & 417.0833 & 1.4 & $285.0403 ; 151.0036 ; 107.0139$ & 2 \\
\hline
\end{tabular}


Table 2. Cont.

\begin{tabular}{|c|c|c|c|c|c|c|c|}
\hline Id & Name & Rt (min) & $\begin{array}{l}\text { Proposed } \\
\text { Formula }\end{array}$ & $\begin{array}{l}\text { Experimental } \\
\mathrm{m} / \mathrm{z}\end{array}$ & $\Delta(\mathrm{ppm})$ & $\begin{array}{l}\text { Diagnostic Product Ions } \\
(\mathrm{m} / \mathrm{z})\end{array}$ & c. 1. \\
\hline 75 & Isorhamnetin 3-O-hexoside & 14.71 & $\mathrm{C}_{22} \mathrm{H}_{22} \mathrm{O}_{12}$ & 477.1049 & 2.3 & $\begin{array}{c}\text { 315.0512; 314.0433; } 300.0275 \\
\text { 271.0244; } 151.0036\end{array}$ & 2 \\
\hline 76 & Kaempferol $O$-pentoside & 14.74 & $\mathrm{C}_{20} \mathrm{H}_{18} \mathrm{O}_{10}$ & 417.0834 & 1.6 & $285.0403 ; 151.0036 ; 107.0139$ & 2 \\
\hline 77 & Phloretin O-hexoside & 14.95 & $\mathrm{C}_{21} \mathrm{H}_{24} \mathrm{O}_{10}$ & 435.1310 & 3.0 & $273.0771 ; 167.0350 ; 123.0452$ & 2 \\
\hline 78 & Myricetin isomer O-hexoside & 15.17 & $\mathrm{C}_{21} \mathrm{H}_{20} \mathrm{O}_{13}$ & 479.0846 & 3.1 & $\begin{array}{c}\text { 317.0303; 271.0259; } 178.9985 \\
\text { 151.0036; } 137.0244\end{array}$ & 2 \\
\hline 79 & Quercetin $O$-acetylhexoside & 15.23 & $\mathrm{C}_{23} \mathrm{H}_{22} \mathrm{O}_{13}$ & 507.1140 & 1.3 & $\begin{array}{c}\mathrm{ESI}(+): 303.0502 ; 165.0186 \\
\text { 153.0185; } 137.0237\end{array}$ & 2 \\
\hline 80 & Diosmetin $O$-hexoside & 15.30 & $\mathrm{C}_{22} \mathrm{H}_{22} \mathrm{O}_{11}$ & 463.1248 & 2.8 & $\begin{array}{l}\text { ESI(+):301.0715; } 286.0479 ; \\
258.0530 ; 153.0185\end{array}$ & 2 \\
\hline 81 & Kaempferol 3-O-pentoside & 15.46 & $\mathrm{C}_{20} \mathrm{H}_{18} \mathrm{O}_{10}$ & 417.0832 & 1.2 & $\begin{array}{c}285.0403 ; 284.0327 ; 151.0036 \\
107.0139\end{array}$ & 2 \\
\hline 82 & Quercetin O-hexoside & 15.56 & $\mathrm{C}_{21} \mathrm{H}_{20} \mathrm{O}_{12}$ & 463.0894 & 2.6 & $\begin{array}{c}301.0354 ; 178.9985 ; 151.0036 \\
121.0295\end{array}$ & 2 \\
\hline 83 & Luteolin O-glucuronide & 15.67 & $\mathrm{C}_{21} \mathrm{H}_{18} \mathrm{O}_{12}$ & 461.0741 & 3.3 & $\begin{array}{c}\text { 285.0407; 243.0296; 241.0502; } \\
\text { 151.0036; } 133.0295\end{array}$ & 2 \\
\hline 84 & Eriodictyol & 15.86 & $\mathrm{C}_{15} \mathrm{H}_{12} \mathrm{O}_{6}$ & 287.0568 & 2.5 & $\begin{array}{c}287.0561 ; 177.0194 ; 151.0036 \\
\text { 135.0452; } 107.0139\end{array}$ & 1 \\
\hline 85 & Isorhamnetin 3-O-pentoside & 15.86 & $\mathrm{C}_{21} \mathrm{H}_{20} \mathrm{O}_{11}$ & 447.0937 & 0.9 & $\begin{array}{c}\text { 315.0512; 314.0433; } 300.0275 \\
\text { 271.0244; } 151.0036\end{array}$ & 2 \\
\hline 86 & Naringenin $O$-hexoside & 15.88 & $\mathrm{C}_{21} \mathrm{H}_{22} \mathrm{O}_{10}$ & 433.1149 & 2.0 & $\begin{array}{c}\text { 271.0613; 227.0713; 177.0194; } \\
\text { 151.0036; } 119.0503\end{array}$ & 2 \\
\hline 87 & Kaempferol O-pentoside & 15.90 & $\mathrm{C}_{20} \mathrm{H}_{18} \mathrm{O}_{10}$ & 417.0835 & 1.8 & $285.0403 ; 151.0036 ; 107.0139$ & 2 \\
\hline 88 & Kaempferol O-deoxyhexoside & 16.11 & $\mathrm{C}_{21} \mathrm{H}_{20} \mathrm{O}_{10}$ & 433.1136 & 1.5 & $\begin{array}{c}\text { ESI(+):287.0553; 165.0187; } \\
153.0185 ; 121.0286\end{array}$ & 2 \\
\hline 89 & Isorhamnetin 3-O-pentoside & 16.41 & $\mathrm{C}_{21} \mathrm{H}_{20} \mathrm{O}_{11}$ & 447.0937 & 0.9 & $\begin{array}{c}\text { 315.0512; 314.0437; } 300.0275 \\
\text { 271.0244; } 151.0036\end{array}$ & 2 \\
\hline 90 & $\begin{array}{l}\text { Quercetin } \\
\text { 3-O-malonyldeoxyhexoside }\end{array}$ & 16.64 & $\mathrm{C}_{24} \mathrm{H}_{22} \mathrm{O}_{14}$ & 533.0948 & 2.0 & $\begin{array}{c}\text { 301.0354; 300.0278; } 178.9985 \\
151.0036 ; 121.0295\end{array}$ & 2 \\
\hline 91 & Quercetin & 17.34 & $\mathrm{C}_{15} \mathrm{H}_{10} \mathrm{O}_{7}$ & 301.0353 & -0.1 & $\begin{array}{c}273.0411 ; 245.0454 ; 178.9985 \\
151.0036 ; 121.0295\end{array}$ & 1 \\
\hline 92 & $\begin{array}{l}\text { Quercetin 3-O- } \\
\text { dihydroxybenzoylpentoside }\end{array}$ & 17.68 & $\mathrm{C}_{27} \mathrm{H}_{22} \mathrm{O}_{14}$ & 569.0950 & 2.3 & $\begin{array}{c}\text { 301.0354; 300.0278; } 178.9985 \\
\text { 151.0036; } 121.0295\end{array}$ & 2 \\
\hline 93 & Luteolin & 17.69 & $\mathrm{C}_{15} \mathrm{H}_{10} \mathrm{O}_{6}$ & 285.0412 & 2.7 & $\begin{array}{c}243.0296 ; 241.0502 ; 151.0036 \\
133.0295\end{array}$ & 1 \\
\hline 94 & $\begin{array}{l}\text { Quercetin } \\
\text { 3-O-coumaroylhexoside }\end{array}$ & 18.05 & $\mathrm{C}_{30} \mathrm{H}_{26} \mathrm{O}_{14}$ & 609.1266 & 2.7 & $\begin{array}{l}\text { 463.0883; 301.0354; 300.0278; } \\
178.9985 ; 151.0036 ; 121.0295\end{array}$ & 2 \\
\hline 95 & $\begin{array}{l}\text { Quercetin } \\
\text { 3-O-coumaroylhexoside }\end{array}$ & 18.28 & $\mathrm{C}_{30} \mathrm{H}_{26} \mathrm{O}_{14}$ & 609.1262 & 2.0 & $\begin{array}{l}\text { 463.0883; 301.0354; 300.0278; } \\
\text { 178.9985; 151.0036; } 121.0295\end{array}$ & 2 \\
\hline 96 & Naringenin isomer & 18.41 & $\mathrm{C}_{15} \mathrm{H}_{12} \mathrm{O}_{5}$ & 271.0614 & 0.7 & $\begin{array}{c}\text { 227.0713; 177.0194; 151.0036; } \\
\text { 119.0503; 107.0139 }\end{array}$ & 2 \\
\hline 97 & Naringenin & 18.76 & $\mathrm{C}_{15} \mathrm{H}_{12} \mathrm{O}_{5}$ & 271.0614 & 0.7 & $\begin{array}{c}\text { 227.0713; 177.0194; 151.0036; } \\
\text { 119.0503; 107.0139 }\end{array}$ & 1 \\
\hline 98 & $\begin{array}{l}\text { Quercetin 3-O- } \\
\text { dihydroxybenzoylpentoside }\end{array}$ & 19.06 & $\mathrm{C}_{27} \mathrm{H}_{22} \mathrm{O}_{14}$ & 569.0937 & 0.0 & $\begin{array}{c}\text { 301.0354; 300.0278; } 273.0411 \\
\text { 151.0036; } 121.0295\end{array}$ & 2 \\
\hline 99 & $\begin{array}{l}\text { Quercetin } O \text { - } \\
\text { dihydroxybenzoylpentoside }\end{array}$ & 19.25 & $\mathrm{C}_{27} \mathrm{H}_{22} \mathrm{O}_{14}$ & 569.0943 & 1.2 & $\begin{array}{c}\text { 301.0354; } 178.9985 ; 151.0036 \\
121.0295\end{array}$ & 2 \\
\hline 100 & Hesperetin & 19.56 & $\mathrm{C}_{16} \mathrm{H}_{14} \mathrm{O}_{6}$ & 301.0726 & 2.7 & $\begin{array}{c}\text { 286.0480; 242.0581; 177.0195; } \\
\text { 164.0115; } 151.0036\end{array}$ & 1 \\
\hline 101 & Apigenin & 20.05 & $\mathrm{C}_{15} \mathrm{H}_{10} \mathrm{O}_{5}$ & 269.0460 & 1.6 & $\begin{array}{c}\text { 225.0557; 151.0036; 117.0346; } \\
107.0139\end{array}$ & 1 \\
\hline 102 & Kaempferol & 20.23 & $\mathrm{C}_{15} \mathrm{H}_{10} \mathrm{O}_{6}$ & 285.0409 & 1.4 & $\begin{array}{c}257.0457 ; 229.0504 ; 151.0036 \\
107.0139\end{array}$ & 1 \\
\hline 103 & Diosmetin & 20.96 & $\mathrm{C}_{16} \mathrm{H}_{12} \mathrm{O}_{6}$ & 299.0567 & 2.1 & $\begin{array}{c}284.0327 ; 257.0411 ; 255.0303 \\
151.0036 ; 107.0139\end{array}$ & 1 \\
\hline 104 & Isorhamnetin & 21.13 & $\mathrm{C}_{16} \mathrm{H}_{12} \mathrm{O}_{7}$ & 315.0517 & 2.1 & $\begin{array}{c}300.0275 ; 271.0244 ; 255.0296 \\
151.0036 ; 107.0139\end{array}$ & 1 \\
\hline 105 & Chrysin & 25.50 & $\mathrm{C}_{15} \mathrm{H}_{10} \mathrm{O}_{4}$ & 253.0508 & 0.8 & $209.0608 ; 151.0036 ; 107.0139$ & 2 \\
\hline
\end{tabular}


Table 3. Retention times (Rt, min), proposed formulas, experimental $m / z$, accuracy $(\Delta, \mathrm{ppm})$, main diagnostic experimental product ions, and confidence level of the identification (c. 1.) of the 108 tentatively identified phenolic acid in Disterigma alaternoides extract in $\operatorname{ESI}(-)$.

\begin{tabular}{|c|c|c|c|c|c|c|c|}
\hline ID & Name & Rt (min) & $\begin{array}{l}\text { Proposed } \\
\text { Formula }\end{array}$ & $\begin{array}{l}\text { Experimental } \\
\mathrm{m} / \mathrm{z}\end{array}$ & $\Delta(\mathrm{ppm})$ & Main Product Ions $(\mathrm{m} / \mathrm{z})$ & c. 1 . \\
\hline 106 & Quinic acid & 0.50 & $\mathrm{C}_{7} \mathrm{H}_{12} \mathrm{O}_{6}$ & 191.0555 & -3.3 & $\begin{array}{c}127.0401 ; 111.0088 ; 87.0084 \\
85.0292\end{array}$ & 2 \\
\hline 107 & Gallic acid & 0.64 & $\mathrm{C}_{7} \mathrm{H}_{6} \mathrm{O}_{5}$ & 169.0145 & 1.4 & $169.0142 ; 125.0243$ & 2 \\
\hline 108 & Methylgallic acid & 0.79 & $\mathrm{C}_{8} \mathrm{H}_{8} \mathrm{O}_{5}$ & 183.0300 & 0.4 & $183.0300 ; 139.0302$ & 2 \\
\hline 109 & Hydroxybenzoyl hexose (I) & 0.82 & $\mathrm{C}_{13} \mathrm{H}_{16} \mathrm{O}_{8}$ & 299.0776 & 1.2 & $137.0244 ; 93.0343$ & 2 \\
\hline 110 & Methyldihydroxybenzoic acid & 1.04 & $\mathrm{C}_{8} \mathrm{H}_{8} \mathrm{O}_{4}$ & 167.0352 & 1.3 & $167.0350 ; 123.0453$ & 2 \\
\hline 111 & Dihydroxybenzoyl hexose (I) & 1.08 & $\mathrm{C}_{13} \mathrm{H}_{16} \mathrm{O}_{9}$ & 315.0727 & 1.6 & 315.0727; 153.0194; 109.0296 & 2 \\
\hline 112 & Dihydroxybenzoic acid & 1.19 & $\mathrm{C}_{7} \mathrm{H}_{6} \mathrm{O}_{4}$ & 153.0196 & 1.8 & $153.0194 ; 109.0296$ & 2 \\
\hline 113 & Phloroglucinol carboxylic acid & 1.22 & $\mathrm{C}_{7} \mathrm{H}_{6} \mathrm{O}_{5}$ & 169.0145 & 1.4 & $169.0142 ; 151.0036 ; 125.0244$ & 2 \\
\hline 114 & Hexosyl caffeoyl hexose (I) & 1.24 & $\mathrm{C}_{21} \mathrm{H}_{28} \mathrm{O}_{14}$ & 503.1415 & 1.6 & $\begin{array}{c}341.0881 ; 179.0350 ; 161.0245 \\
135.0453\end{array}$ & 2 \\
\hline 115 & Hydroxybenzoyl hexose (II) & 1.29 & $\mathrm{C}_{13} \mathrm{H}_{16} \mathrm{O}_{8}$ & 299.0777 & 1.4 & $299.0777 ; 137.0244 ; 93.0343$ & 2 \\
\hline 116 & Methlhydroxybenzoyl hexose & 1.38 & $\mathrm{C}_{14} \mathrm{H}_{18} \mathrm{O}_{8}$ & 313.0936 & 2.2 & 151.0401; 107.0502 & 2 \\
\hline 117 & Dihydroxybenzoyl hexose (II) & 1.43 & $\mathrm{C}_{13} \mathrm{H}_{16} \mathrm{O}_{9}$ & 315.0724 & 0.8 & $315.0727 ; 153.0194 ; 109.0296$ & 2 \\
\hline 118 & Hydroxybenzoyl hexose (III) & 1.62 & $\mathrm{C}_{13} \mathrm{H}_{16} \mathrm{O}_{8}$ & 299.0777 & 1.6 & 299.0777; 137.0244; 93.0343 & 2 \\
\hline 119 & Neochlorogenic acid & 1.68 & $\mathrm{C}_{16} \mathrm{H}_{18} \mathrm{O}_{9}$ & 353.0884 & 1.6 & $\begin{array}{c}191.0562 ; 179.0350 ; 145.0452 ; \\
135.0453\end{array}$ & 2 \\
\hline 120 & Dihydroxybenzoyl pentose & 1.72 & $\mathrm{C}_{12} \mathrm{H}_{14} \mathrm{O}_{8}$ & 285.0621 & 1.7 & $285.0618 ; 153.0194 ; 109.0296$ & 2 \\
\hline 121 & Chlorogenoyl hexose & 1.91 & $\mathrm{C}_{22} \mathrm{H}_{28} \mathrm{O}_{14}$ & 515.1409 & 0.6 & $\begin{array}{c}353.0883 ; 191.0561 ; 179.0349 \\
135.0453\end{array}$ & 2 \\
\hline 122 & Hexosyl caffeoyl hexose (I) & 2.07 & $\mathrm{C}_{21} \mathrm{H}_{28} \mathrm{O}_{14}$ & 503.1418 & 2.3 & $\begin{array}{c}341.0881 ; 179.0350 ; 161.0245 \\
135.0453\end{array}$ & 2 \\
\hline 123 & Caffeoyl hexose (I) & 2.10 & $\mathrm{C}_{15} \mathrm{H}_{18} \mathrm{O}_{9}$ & 341.0883 & 1.3 & $341.0881 ; 179.0350 ; 135.0453$ & 2 \\
\hline 124 & Coumaroyl hexose (I) & 2.10 & $\mathrm{C}_{15} \mathrm{H}_{18} \mathrm{O}_{8}$ & 325.0933 & 1.3 & $325.0933 ; 163.0402 ; 119.0503$ & 2 \\
\hline 125 & Dihydroxybenzoyl hexose (III) & 2.14 & $\mathrm{C}_{13} \mathrm{H}_{16} \mathrm{O}_{9}$ & 315.0725 & 1.1 & 315.0727; 153.0194; 109.0296 & 2 \\
\hline 126 & Dihydroxybenzoyl hexose (IV) & 2.37 & $\mathrm{C}_{13} \mathrm{H}_{16} \mathrm{O}_{9}$ & 315.0725 & 1.1 & $315.0727 ; 153.0194 ; 109.0296$ & 2 \\
\hline 127 & Hydroxyferuloyl hexose (I) & 2.74 & $\mathrm{C}_{16} \mathrm{H}_{20} \mathrm{O}_{10}$ & 371.0988 & 1.2 & $\begin{array}{c}209.0456 ; 191.0352 ; 147.0452 \\
119.0503\end{array}$ & 2 \\
\hline 128 & Quinoyl coumaric acid & 2.76 & $\mathrm{C}_{16} \mathrm{H}_{18} \mathrm{O}_{8}$ & 337.0935 & 1.7 & $\begin{array}{c}191.0561 ; 173.0459 ; 163.0400 \\
119.0502\end{array}$ & 2 \\
\hline 129 & Caffeoyl hexose (II) & 3.21 & $\mathrm{C}_{15} \mathrm{H}_{18} \mathrm{O}_{9}$ & 341.0880 & 0.7 & 179.0350; 135.0453 & 2 \\
\hline 130 & Caffeoyl hexose (III) & 3.49 & $\mathrm{C}_{15} \mathrm{H}_{18} \mathrm{O}_{9}$ & 341.0879 & 0.2 & $179.0350 ; 161.0244 ; 135.0453$ & 2 \\
\hline 131 & Caffeic acid & 3.58 & $\mathrm{C}_{9} \mathrm{H}_{8} \mathrm{O}_{4}$ & 179.0352 & 1.2 & $179.0350 ; 135.0453$ & 2 \\
\hline 132 & Feruloyl hexose (I) & 3.60 & $\mathrm{C}_{16} \mathrm{H}_{20} \mathrm{O}_{9}$ & 355.1040 & 1.5 & $\begin{array}{c}193.0507 ; 178.0272 ; 149.0608 ; \\
134.0374\end{array}$ & 2 \\
\hline 133 & Coumaroyl hexose (II) & 3.61 & $\mathrm{C}_{15} \mathrm{H}_{18} \mathrm{O}_{8}$ & 325.0934 & 1.5 & $325.0933 ; 163.0402 ; 119.0503$ & 2 \\
\hline 134 & Chlorogenic acid & 3.76 & $\mathrm{C}_{16} \mathrm{H}_{18} \mathrm{O}_{9}$ & 353.0880 & 0.4 & $\begin{array}{c}353.0879 ; 191.0562 ; 179.0350 \\
145.0452\end{array}$ & 2 \\
\hline 135 & Hydroxyferuloyl hexose (II) & 4.04 & $\mathrm{C}_{16} \mathrm{H}_{20} \mathrm{O}_{10}$ & 371.0988 & 1.2 & $\begin{array}{c}\text { 209.0456; } 191.0352 ; 147.0452 ; \\
119.0503\end{array}$ & 2 \\
\hline 136 & Hydroxyferulic acid & 4.82 & $\mathrm{C}_{10} \mathrm{H}_{10} \mathrm{O}_{5}$ & 209.0458 & 1.1 & $\begin{array}{l}191.0352 ; 147.0452 ; 119.0503 \\
223.0611 ; 208.0377 ; 193.0141 ;\end{array}$ & 2 \\
\hline 137 & Sinapoyl hexose (I) & 5.23 & $\mathrm{C}_{17} \mathrm{H}_{22} \mathrm{O}_{10}$ & 385.1145 & 1.2 & $\begin{array}{c}179.0913 ; 164.0478 ; 149.0243 ; \\
121.0295\end{array}$ & 2 \\
\hline 138 & Feruloyl hexose (II) & 5.68 & $\mathrm{C}_{16} \mathrm{H}_{20} \mathrm{O}_{9}$ & 355.1037 & 0.7 & $\begin{array}{c}193.0507 ; 178.0272 ; 149.0608 ; \\
134.0374\end{array}$ & 2 \\
\hline 139 & Chlorogenic acid isomer & 5.93 & $\mathrm{C}_{16} \mathrm{H}_{18} \mathrm{O}_{9}$ & 353.0886 & 2.3 & $\begin{array}{c}353.0879 ; 191.0562 ; 179.0350 \\
145.0452\end{array}$ & 2 \\
\hline 140 & Coumaric acid (I) & 6.06 & $\mathrm{C}_{9} \mathrm{H}_{8} \mathrm{O}_{3}$ & 163.0404 & 2.0 & $163.0402 ; 119.0503$ & 2 \\
\hline 141 & Coumaroyl quinic acid (I) & 6.19 & $\mathrm{C}_{16} \mathrm{H}_{18} \mathrm{O}_{8}$ & 337.0936 & 2.0 & $\begin{array}{c}\text { 191.0560; } 173.0455 ; 163.0401 ; \\
119.0501\end{array}$ & 2 \\
\hline 142 & Caffeoyl shiikimoyl hexose (I) & 6.46 & $\mathrm{C}_{22} \mathrm{H}_{26} \mathrm{O}_{13}$ & 497.1307 & 1.3 & $335.0773 ; 179.0349 ; 135.0451$ & 2 \\
\hline 143 & Hydroxyferulic acid isomer & 6.61 & $\mathrm{C}_{10} \mathrm{H}_{10} \mathrm{O}_{5}$ & 209.0458 & 1.1 & $\begin{array}{c}209.0456 ; 165.0557 ; 123.0452 ; \\
81.0342\end{array}$ & 2 \\
\hline 144 & Coumaric acid (II) & 6.69 & $\mathrm{C}_{9} \mathrm{H}_{8} \mathrm{O}_{3}$ & 163.0404 & 2.1 & $163.0402 ; 119.0503$ & 2 \\
\hline 145 & Acetyl dihydroxybenzoic acid & 6.71 & $\mathrm{C}_{9} \mathrm{H}_{8} \mathrm{O}_{5}$ & 195.0301 & 1.2 & $\begin{array}{l}195.0299 ; 153.0194 ; 109.0296 \\
223.0611 ; 208.0377 ; 193.0141\end{array}$ & 2 \\
\hline 146 & Sinapoyl hexose (II) & 7.06 & $\mathrm{C}_{17} \mathrm{H}_{22} \mathrm{O}_{10}$ & 385.1146 & 1.4 & $\begin{array}{c}179.0913 ; 164.0478 ; 149.0243 \\
121.0295\end{array}$ & 2 \\
\hline 147 & Caffeoyl shiikimic acid (I) & 7.15 & $\mathrm{C}_{16} \mathrm{H}_{16} \mathrm{O}_{8}$ & 335.0777 & 1.3 & $\begin{array}{c}179.0349 ; 173.0452 ; 161.0242 \\
135.0451\end{array}$ & 2 \\
\hline 148 & Coumaroyl hexose (III) & 7.35 & $\mathrm{C}_{15} \mathrm{H}_{18} \mathrm{O}_{8}$ & 325.0936 & 2.1 & $325.0933 ; 163.0402 ; 119.0503$ & 2 \\
\hline 149 & Feruloyl quinic acid & 7.88 & $\mathrm{C}_{17} \mathrm{H}_{20} \mathrm{O}_{9}$ & 367.1041 & 1.8 & $\begin{array}{l}\text { 193.0507; 191.0560; 178.0272; } \\
173.0455 ; 149.0608 ; 134.0374\end{array}$ & 2 \\
\hline
\end{tabular}


Table 3. Cont.

\begin{tabular}{|c|c|c|c|c|c|c|c|}
\hline ID & Name & Rt (min) & $\begin{array}{c}\text { Proposed } \\
\text { Formula }\end{array}$ & $\begin{array}{l}\text { Experimental } \\
\mathrm{m} / \mathrm{z}\end{array}$ & $\Delta(\mathrm{ppm})$ & Main Product Ions $(\mathrm{m} / \mathrm{z})$ & c. 1 . \\
\hline 150 & Coumaroyl quinic acid (II) & 8.11 & $\mathrm{C}_{16} \mathrm{H}_{18} \mathrm{O}_{8}$ & 337.0936 & 2.0 & $\begin{array}{c}\text { 191.0560; } 173.0455 ; 163.0401 \\
119.0501\end{array}$ & 2 \\
\hline 151 & Ferulic acid & 8.16 & $\mathrm{C}_{10} \mathrm{H}_{10} \mathrm{O}_{4}$ & 193.0509 & 1.5 & $178.0272 ; 149.0608 ; 134.0374$ & 1 \\
\hline 152 & Feruloyl shiikimoyl hexose & 8.25 & $\mathrm{C}_{23} \mathrm{H}_{28} \mathrm{O}_{13}$ & 511.1468 & 2.1 & $\begin{array}{c}\text { 193.0507; } 178.0272 ; 149.0608 ; \\
134.0374\end{array}$ & 2 \\
\hline 153 & Caffeoyl hexosyl arbutin (I) & 8.93 & $\mathrm{C}_{27} \mathrm{H}_{32} \mathrm{O}_{15}$ & 595.1683 & 2.4 & $\begin{array}{c}\text { 433.1143; 323.0779; } 179.0348 \\
\text { 161.0245; } 135.0454\end{array}$ & 2 \\
\hline 154 & Caffeoyl shiikimic acid (II) & 9.01 & $\mathrm{C}_{16} \mathrm{H}_{16} \mathrm{O}_{8}$ & 335.0780 & 2.3 & $\begin{array}{c}179.0349 ; 173.0452 ; 161.0242 ; \\
135.0451\end{array}$ & 2 \\
\hline 155 & Caffeoyl shiikimoyl hexose (II) & 9.29 & $\mathrm{C}_{22} \mathrm{H}_{26} \mathrm{O}_{13}$ & 497.1310 & 1.8 & $\begin{array}{c}335.0773 ; 179.0349 ; 161.0245 \\
135.0451\end{array}$ & 2 \\
\hline 156 & Sinapic acid & 9.33 & $\mathrm{C}_{11} \mathrm{H}_{12} \mathrm{O}_{5}$ & 223.0617 & 2.4 & $\begin{array}{l}\text { 208.0377; 193.0141; 179.0913; } \\
\text { 164.0478; 149.0243; } 121.0295\end{array}$ & 2 \\
\hline 157 & Coumaroyl shiikimic acid & 9.65 & $\mathrm{C}_{16} \mathrm{H}_{16} \mathrm{O}_{7}$ & 319.0829 & 1.8 & $\begin{array}{c}173.0454 ; 163.0402 ; 155.0350 ; \\
119.0503\end{array}$ & 2 \\
\hline 158 & Acetyl caffeoyl deoxyhexoside & 9.91 & $\mathrm{C}_{17} \mathrm{H}_{20} \mathrm{O}_{9}$ & 367.1037 & 0.6 & $179.0350 ; 161.0244 ; 135.0452$ & 2 \\
\hline 159 & Caffeoyl hexosyl arbutin (II) & 9.95 & $\mathrm{C}_{27} \mathrm{H}_{32} \mathrm{O}_{15}$ & 595.1683 & 2.4 & $\begin{array}{c}433.1143 ; 323.0779 ; 179.0348 ; \\
135.0454\end{array}$ & 2 \\
\hline 160 & Caffeoyl hexosyl arbutin (III) & 10.05 & $\mathrm{C}_{27} \mathrm{H}_{32} \mathrm{O}_{15}$ & 595.1686 & 2.9 & $\begin{array}{c}433.1143 ; 323.0779 ; 179.0348 ; \\
135.0454\end{array}$ & 2 \\
\hline 161 & Hydroxybenzoyl arbutin (I) & 10.12 & $\mathrm{C}_{19} \mathrm{H}_{20} \mathrm{O}_{9}$ & 391.1044 & 2.4 & $\begin{array}{c}281.0669 ; 137.0245 ; 109.0296 ; \\
93.0344\end{array}$ & 2 \\
\hline 162 & $\begin{array}{l}\text { Caffeoyl hexosyl } \\
\text { trihydroxymethoxyphenyl } \\
\text { propanoic acid }\end{array}$ & 10.16 & $\mathrm{C}_{25} \mathrm{H}_{28} \mathrm{O}_{14}$ & 551.1416 & 1.8 & $\begin{array}{c}\text { 389.0873; 345.0975; 327.0873; } \\
\text { 179.0349; 165.0557; } 161.0243 ; \\
\text { 135.0451; } 121.0296\end{array}$ & 2 \\
\hline 163 & Caffeoyl arbutin (I) & 11.05 & $\mathrm{C}_{21} \mathrm{H}_{22} \mathrm{O}_{10}$ & 433.1139 & -0.3 & $\begin{array}{l}\text { 323.0778; 179.0351; 161.0244; } \\
135.0453 ; 133.0295 ; 109.0295\end{array}$ & 2 \\
\hline 164 & Galloyl valeryl hexoside & 11.26 & $\mathrm{C}_{18} \mathrm{H}_{24} \mathrm{O}_{11}$ & 415.1255 & 2.2 & 169.0142; 125.0244 & 2 \\
\hline 165 & Caffeoyl arbutin (II) & 11.35 & $\mathrm{C}_{21} \mathrm{H}_{22} \mathrm{O}_{10}$ & 433.1139 & -0.3 & $\begin{array}{l}\text { 323.0778; 179.0351; 161.0244; } \\
\text { 135.0453; 133.0295; } 109.0295\end{array}$ & 2 \\
\hline 166 & Ferulic acid isomer & 11.85 & $\mathrm{C}_{10} \mathrm{H}_{10} \mathrm{O}_{4}$ & 193.0509 & 1.5 & $175.0402 ; 149.0608$ & 2 \\
\hline 167 & Coumaroyl iridoid (I) & 11.98 & $\mathrm{C}_{25} \mathrm{H}_{28} \mathrm{O}_{13}$ & 535.1460 & 0.6 & $\begin{array}{c}191.0350 ; 163.0400 ; 147.0452 ; \\
119.0502\end{array}$ & 2 \\
\hline 168 & Coumaroyl iridoid (II) & 12.04 & $\mathrm{C}_{25} \mathrm{H}_{28} \mathrm{O}_{13}$ & 535.1460 & 0.6 & $\begin{array}{c}191.0350 ; 163.0400 ; 147.0452 ; \\
119.0502\end{array}$ & 2 \\
\hline 169 & Caffeoyl methoxyarbutin & 12.31 & $\mathrm{C}_{22} \mathrm{H}_{24} \mathrm{O}_{11}$ & 463.1249 & 0.7 & $\begin{array}{l}323.0771 ; 179.0349 ; 161.0244 ; \\
139.0401 ; 135.0453 ; 124.0166\end{array}$ & 2 \\
\hline 170 & Coumaroyl coumaric acid & 12.55 & $\mathrm{C}_{18} \mathrm{H}_{14} \mathrm{O}_{5}$ & 309.0776 & 2.4 & $163.0401 ; 119.0503$ & 2 \\
\hline 171 & $\begin{array}{l}\text { Caffeoyl dihydroxybenzoyl } \\
\text { hexose }\end{array}$ & 13.63 & $\mathrm{C}_{22} \mathrm{H}_{22} \mathrm{O}_{12}$ & 477.1046 & 1.6 & $\begin{array}{c}\text { 315.0724; 179.0347; 153.0193; } \\
\text { 135.0451; 109.0296 }\end{array}$ & 2 \\
\hline 172 & Dicaffeoyl hexoside & 14.36 & $\mathrm{C}_{24} \mathrm{H}_{24} \mathrm{O}_{12}$ & 503.1213 & 3.6 & $\begin{array}{c}341.0875 ; 323.0778 ; 179.0351 \\
135.0453\end{array}$ & 2 \\
\hline 173 & $\begin{array}{l}\text { Dihydroxybenzoyl valeryl } \\
\text { hexose (I) }\end{array}$ & 14.44 & $\mathrm{C}_{18} \mathrm{H}_{24} \mathrm{O}_{10}$ & 399.1303 & 1.5 & $153.0193 ; 109.0294$ & 2 \\
\hline 174 & $\begin{array}{l}\text { Coumaroyl dihydroxybenzoyl } \\
\text { hexose (II) }\end{array}$ & 14.81 & $\mathrm{C}_{22} \mathrm{H}_{22} \mathrm{O}_{11}$ & 461.1101 & 2.6 & $315.0741 ; 153.0195 ; 109.0296$ & 2 \\
\hline 175 & $\begin{array}{l}\text { Dihydroxybenzoyl valeryl } \\
\text { hexose (II) }\end{array}$ & 14.95 & $\mathrm{C}_{18} \mathrm{H}_{24} \mathrm{O}_{10}$ & 399.1303 & 1.5 & $399.1290 ; 153.0193 ; 109.0294$ & 2 \\
\hline 176 & Coumaroyl coumaric acid & 15.14 & $\mathrm{C}_{18} \mathrm{H}_{14} \mathrm{O}_{5}$ & 309.0777 & 2.7 & $309.0776 ; 163.0401 ; 119.0503$ & 2 \\
\hline 177 & $\begin{array}{l}\text { Dihydroxybenzoyl benzoyl } \\
\text { hexose (I) }\end{array}$ & 15.15 & $\mathrm{C}_{20} \mathrm{H}_{20} \mathrm{O}_{10}$ & 419.0991 & 1.8 & $315.0727 ; 153.0194 ; 109.0296$ & 2 \\
\hline 178 & $\begin{array}{l}\text { Coumaroyl hydroxybenzoyl } \\
\text { hexose }\end{array}$ & 15.24 & $\mathrm{C}_{22} \mathrm{H}_{22} \mathrm{O}_{10}$ & 445.1146 & 1.2 & $\begin{array}{l}\text { 307.0822; 163.0403; 145.0294; } \\
\text { 137.0243; 119.0501; } 93.0343 \\
\text { 531.1481; 307.0823; 223.0612; }\end{array}$ & 2 \\
\hline 179 & Sinapoyl coumaroyl hexose & 15.31 & $\mathrm{C}_{26} \mathrm{H}_{28} \mathrm{O}_{12}$ & 531.1520 & 2.3 & $\begin{array}{c}208.0378 ; 193.0141 ; 163.0400 \\
149.0243 ; 119.0503\end{array}$ & 2 \\
\hline 180 & Hydroxybenzoyl arbutin (II) & 15.52 & $\mathrm{C}_{19} \mathrm{H}_{20} \mathrm{O}_{9}$ & 391.1044 & 2.5 & $\begin{array}{c}391.1047 ; 281.0669 ; 137.0244 ; \\
93.0343\end{array}$ & 2 \\
\hline 181 & $\begin{array}{l}\text { Dihydroxybenzoyl benzoyl } \\
\text { hexose (II) }\end{array}$ & 15.58 & $\mathrm{C}_{20} \mathrm{H}_{20} \mathrm{O}_{10}$ & 419.0994 & 2.5 & $315.0727 ; 153.0194 ; 109.0296$ & 2 \\
\hline 182 & Caffeoyl acetyl arbutin (I) & 15.71 & $\mathrm{C}_{23} \mathrm{H}_{24} \mathrm{O}_{11}$ & 475.1246 & 0.0 & $\begin{array}{c}179.0348 ; 161.0244 ; 135.0452 ; \\
133.0295\end{array}$ & 2 \\
\hline 183 & Caffeoyl acetyl arbutin (II) & 15.84 & $\mathrm{C}_{23} \mathrm{H}_{24} \mathrm{O}_{11}$ & 475.1246 & 0.0 & $\begin{array}{c}179.0348 ; 161.0244 ; 135.0452 ; \\
133.0295\end{array}$ & 2 \\
\hline 184 & Chlorogenic acid derivative (I) & 15.85 & $\mathrm{C}_{26} \mathrm{H}_{28} \mathrm{O}_{12}$ & 531.1516 & 1.5 & $\begin{array}{c}353.0879 ; 191.0562 ; 179.0350 \\
145.0452\end{array}$ & 3 \\
\hline
\end{tabular}


Table 3. Cont.

\begin{tabular}{|c|c|c|c|c|c|c|c|}
\hline ID & Name & Rt (min) & $\begin{array}{l}\text { Proposed } \\
\text { Formula }\end{array}$ & $\begin{array}{l}\text { Experimental } \\
\mathrm{m} / \mathrm{z}\end{array}$ & $\Delta(\mathrm{ppm})$ & Main Product Ions $(\mathrm{m} / \mathrm{z})$ & c. 1 . \\
\hline 185 & $\begin{array}{l}\text { Hydroxybenzoyl benzoyl } \\
\text { hexose (I) }\end{array}$ & 15.86 & $\mathrm{C}_{20} \mathrm{H}_{20} \mathrm{O}_{9}$ & 403.1080 & 2.0 & $137.0244 ; 93.0344$ & 2 \\
\hline 186 & Caffeoyl coumaroyl hexose & 16.06 & $\mathrm{C}_{24} \mathrm{H}_{24} \mathrm{O}_{11}$ & 487.1257 & 2.2 & $\begin{array}{c}323.0772 ; 179.0349 ; 163.0399 \\
119.0501\end{array}$ & 2 \\
\hline 187 & $\begin{array}{l}\text { Dihydroxybenzoyl valeryl } \\
\text { hexose (III) }\end{array}$ & 16.32 & $\mathrm{C}_{18} \mathrm{H}_{24} \mathrm{O}_{10}$ & 399.1303 & 1.5 & $153.0193 ; 109.0294$ & 2 \\
\hline 188 & Caffeoyl feruloyl hexose (I) & 16.43 & $\mathrm{C}_{25} \mathrm{H}_{26} \mathrm{O}_{12}$ & 517.1358 & 1.5 & $\begin{array}{c}337.0929 ; 179.0349 ; 175.0399 ; \\
135.0452\end{array}$ & 2 \\
\hline 189 & $\begin{array}{l}\text { Coumaroyl dihydroxybenzoyl } \\
\text { hexose (I) }\end{array}$ & 16.46 & $\mathrm{C}_{22} \mathrm{H}_{22} \mathrm{O}_{11}$ & 461.1101 & 2.5 & $315.0741 ; 153.0195 ; 109.0296$ & 2 \\
\hline 190 & $\begin{array}{l}\text { Dihydroxybenzoyl } \\
\text { dihydroxybenzoic acid }\end{array}$ & 16.60 & $\mathrm{C}_{14} \mathrm{H}_{10} \mathrm{O}_{7}$ & 289.0362 & 2.8 & $153.0194 ; 109.0296$ & 2 \\
\hline 191 & Caffeoyl feruloyl hexose (II) & 16.64 & $\mathrm{C}_{25} \mathrm{H}_{26} \mathrm{O}_{12}$ & 517.1358 & 1.5 & $179.0349 ; 175.0399 ; 135.0452$ & 2 \\
\hline 192 & Dicoumaroyl hexose (I) & 16.87 & $\mathrm{C}_{24} \mathrm{H}_{24} \mathrm{O}_{10}$ & 471.1305 & 1.8 & $\begin{array}{c}\text { 307.0826; } 163.0400 ; 145.0294 \\
119.0502\end{array}$ & 2 \\
\hline 193 & $\begin{array}{l}\text { Feruloyl dihydroxybenzoyl } \\
\text { hexose }\end{array}$ & 17.19 & $\mathrm{C}_{23} \mathrm{H}_{24} \mathrm{O}_{12}$ & 491.1204 & 1.8 & $\begin{array}{c}\text { 315.0724; } 193.0505 ; 175.0401 ; \\
\text { 160.0165; 153.0192; 134.0371; } \\
\text { 109.0294 }\end{array}$ & 2 \\
\hline 194 & Coumaroyl Feruloyl hexose (I) & 17.28 & $\mathrm{C}_{25} \mathrm{H}_{26} \mathrm{O}_{11}$ & 501.1413 & 2.0 & $\begin{array}{c}\text { 337.0936; 307.0824; 193.0507; } \\
\text { 178.0272; 163.0400; 149.0608; } \\
\text { 134.0374; } 119.0502\end{array}$ & 2 \\
\hline 195 & Caffeoyl valeryl hexose (I) & 17.32 & $\mathrm{C}_{20} \mathrm{H}_{26} \mathrm{O}_{10}$ & 425.1459 & 1.3 & $179.0350 ; 135.0451$ & 3 \\
\hline 196 & Caffeoyl valeryl hexose (II) & 17.39 & $\mathrm{C}_{20} \mathrm{H}_{26} \mathrm{O}_{10}$ & 425.1457 & 0.9 & $179.0350 ; 135.0451$ & 3 \\
\hline 197 & Caffeoyl benzoyl hexose (I) & 17.40 & $\mathrm{C}_{22} \mathrm{H}_{22} \mathrm{O}_{10}$ & 445.1146 & 1.3 & $179.0345 ; 135.0452 ; 121.0296$ & 2 \\
\hline 198 & Diferuloyl hexose & 17.61 & $\mathrm{C}_{26} \mathrm{H}_{28} \mathrm{O}_{12}$ & 531.1521 & 2.4 & $\begin{array}{c}\text { 337.0936; } 193.0507 ; 178.0272 ; \\
134.0374\end{array}$ & 2 \\
\hline 199 & Caffeoyl benzoyl hexose (II) & 17.72 & $\mathrm{C}_{22} \mathrm{H}_{22} \mathrm{O}_{10}$ & 445.1149 & 1.9 & $179.0345 ; 135.0452$ & 2 \\
\hline 200 & $\begin{array}{l}\text { Hydroxybenzoyl benzoyl } \\
\text { hexose (II) }\end{array}$ & 17.84 & $\mathrm{C}_{20} \mathrm{H}_{20} \mathrm{O}_{9}$ & 403.1080 & 2.0 & $137.0244 ; 93.0344$ & 2 \\
\hline 201 & Dicaffeoyl shiikimic acid (II) & 17.94 & $\mathrm{C}_{25} \mathrm{H}_{22} \mathrm{O}_{11}$ & 497.1080 & -1.9 & $179.0350 ; 161.0243 ; 135.0451$ & 2 \\
\hline 202 & $\begin{array}{l}\text { Coumaroyl } \\
\text { methylhydroxybenzoyl hexose } \\
\text { (I) }\end{array}$ & 17.95 & $\mathrm{C}_{23} \mathrm{H}_{24} \mathrm{O}_{10}$ & 459.1302 & 1.2 & $\begin{array}{c}307.0818 ; 163.0402 ; 145.0295 \\
119.0502\end{array}$ & 2 \\
\hline 203 & Coumaroyl valeryl hexose (I) & 17.96 & $\mathrm{C}_{20} \mathrm{H}_{26} \mathrm{O}_{9}$ & 409.1513 & 2.2 & $163.0399 ; 119.0501$ & 3 \\
\hline 204 & Dicoumaroyl hexose (II) & 18.04 & $\mathrm{C}_{24} \mathrm{H}_{24} \mathrm{O}_{10}$ & 471.1306 & 2.0 & $\begin{array}{c}\text { 307.0820; } 163.0399 ; 145.0293 \\
119.0501\end{array}$ & 2 \\
\hline 205 & Dicaffeoyl shiikimic acid (I) & 18.06 & $\mathrm{C}_{25} \mathrm{H}_{22} \mathrm{O}_{11}$ & 497.1068 & -4.2 & $179.0350 ; 161.0243 ; 135.0451$ & 2 \\
\hline 206 & $\begin{array}{l}\text { Dihydroxybenzoyl benzoyl } \\
\text { hexose (III) }\end{array}$ & 18.36 & $\mathrm{C}_{20} \mathrm{H}_{20} \mathrm{O}_{10}$ & 419.0992 & 1.9 & $153.0194 ; 109.0295$ & 2 \\
\hline 207 & Feruloyl valeryl hexose & 18.38 & $\mathrm{C}_{21} \mathrm{H}_{28} \mathrm{O}_{10}$ & 439.1618 & 1.9 & $\begin{array}{c}\text { 193.0507; } 178.0272 ; 149.0608 ; \\
134.0374\end{array}$ & 2 \\
\hline 208 & $\begin{array}{l}\text { Coumaroyl } \\
\text { methylhydroxybenzoyl hexose } \\
\text { (II) }\end{array}$ & 18.42 & $\mathrm{C}_{23} \mathrm{H}_{24} \mathrm{O}_{10}$ & 459.1302 & 1.2 & $\begin{array}{c}\text { 307.0818; } 163.0402 ; 145.0295 \\
119.0502\end{array}$ & 2 \\
\hline 209 & Coumaroyl Feruloyl hexose (II) & 18.58 & $\mathrm{C}_{25} \mathrm{H}_{26} \mathrm{O}_{11}$ & 501.1413 & 2.0 & $\begin{array}{c}193.0507 ; 175.0401 ; 163.0400 ; \\
\text { 160.0165; 149.0608; 145.0295; } \\
\text { 134.0374; 119.0502 }\end{array}$ & 2 \\
\hline 210 & Chlorogenic acid derivative (II) & 19.14 & $\mathrm{C}_{26} \mathrm{H}_{28} \mathrm{O}_{12}$ & 531.1512 & 0.7 & $\begin{array}{c}353.0879 ; 191.0562 ; 179.0350 \\
145.0452\end{array}$ & 2 \\
\hline 211 & $\begin{array}{l}\text { Caffeoyl dihydroxybenzoyl } \\
\text { mevalonic acid }\end{array}$ & 19.47 & $\mathrm{C}_{22} \mathrm{H}_{22} \mathrm{O}_{10}$ & 445.1149 & 1.9 & $\begin{array}{c}179.0345 ; 153.0194 ; 135.0452 ; \\
109.0296\end{array}$ & 2 \\
\hline 212 & Coumaroyl valeryl hexose (II) & 19.77 & $\mathrm{C}_{20} \mathrm{H}_{26} \mathrm{O}_{9}$ & 409.1512 & 1.8 & 409.1500; 163.0399; 119.0501 & 3 \\
\hline 213 & Caffeoyl cinnamoyl hexose & 20.26 & $\mathrm{C}_{24} \mathrm{H}_{24} \mathrm{O}_{10}$ & 471.1303 & 1.3 & 471.1230; 179.0350; 135.0452 & 2 \\
\hline
\end{tabular}


Table 4. Retention times (Rt, min), proposed formulas, experimental $m / z$, accuracy $(\Delta, \mathrm{ppm})$, main diagnostic experimental product ions, and confidence level of the identification (c. 1.) of the 36 annotated proanthocyanidins in Disterigma alaternoides extract in ESI(-).

\begin{tabular}{|c|c|c|c|c|c|c|c|}
\hline ID & Name & Rt (min) & $\begin{array}{l}\text { Proposed } \\
\text { Formula }\end{array}$ & $\begin{array}{c}\text { Experimental } \\
\mathrm{m} / \mathrm{z}\end{array}$ & $\Delta(\mathrm{ppm})$ & Main Product Ions $(\mathrm{m} / \mathrm{z})$ & c. 1 . \\
\hline 214 & A-Procyanidin tetramer & 2.56 & $\mathrm{C}_{60} \mathrm{H}_{48} \mathrm{O}_{24}$ & 575.1209 & 2.5 & $\begin{array}{c}\text { 981.1918; 863.1859; 829.1519; } \\
\text { 693.1247; 573.1050; 451.1032; } \\
\text { 425.0874; 411.0724; 407.0772; } \\
\text { 289.0720; 285.0406; 137.0245; } \\
\text { 125.0245 }\end{array}$ & 2 \\
\hline 215 & B-Procyanidin dimer & 5.73 & $\mathrm{C}_{30} \mathrm{H}_{26} \mathrm{O}_{12}$ & 577.1365 & 2.3 & $\begin{array}{c}\text { 451.1032; 425.0874; 407.0772; } \\
\text { 289.0720; 287.0568; 245.0828; } \\
\text { 137.02443; 125.0244 }\end{array}$ & 2 \\
\hline 216 & A-Procyanidin pentamer & 5.78 & $\mathrm{C}_{75} \mathrm{H}_{60} \mathrm{O}_{30}$ & 719.1533 & 3.0 & $\begin{array}{l}\text { 981.1981; 863.1859; 711.1358; } \\
\text { 693.1247; 575.1198; 573.1064; } \\
\text { 451.1054; 449.0878; 423.0739; } \\
\text { 411.0740; 407.0772; 289.0720; } \\
\text { 285.0406; 137.0245; } 125.0245\end{array}$ & 2 \\
\hline 217 & B-Procyanidin trimer & 5.94 & $\mathrm{C}_{45} \mathrm{H}_{38} \mathrm{O}_{18}$ & 865.2004 & 2.2 & $\begin{array}{c}\text { 713.1520; } 577.1334 ; 575.1216 ; \\
\text { 451.1032; } 425.0874 ; \text {;07.0772; } \\
\text { 289.0720; } 287.0562 ; 137.0244 ; \\
125.0244\end{array}$ & 2 \\
\hline 218 & B-Procyanidin tetramer & 7.08 & $\mathrm{C}_{60} \mathrm{H}_{50} \mathrm{O}_{24}$ & 576.1287 & 2.4 & $\begin{array}{c}\text { 577.1334; } 575.1216 ; 451.1032 ; \\
425.0874 ; \text { 407.0772; } 289.0720 ; \\
\text { 287.0562; } 245.0828 ; 137.0244 ; \\
125.0244\end{array}$ & 2 \\
\hline 219 & A-Procyanidin trimer & 7.33 & $\mathrm{C}_{45} \mathrm{H}_{36} \mathrm{O}_{18}$ & 863.1846 & 2.0 & $\begin{array}{c}\text { 711.1358; } 693.1247 ; \text { 573.1050; } \\
\text { 451.1032; } 411.0724 ; \text { 407.0772; } \\
\text { 289.0720; } 285.0406 ; 137.0245 ; \\
125.0245\end{array}$ & 2 \\
\hline 220 & B-Procyanidin tetramer & 7.59 & $\mathrm{C}_{60} \mathrm{H}_{50} \mathrm{O}_{24}$ & 576.1291 & 3.0 & $\begin{array}{l}\text { 577.1334; } 575.1216 ; 451.1032 ; \\
\text { 425.0874; } 407.0772 ; 289.0720 ; \\
\text { 287.0562; } 245.0828 ; 137.0244 ; \\
125.0244\end{array}$ & 2 \\
\hline 221 & A-Procyanidin tetramer & 8.14 & $\mathrm{C}_{60} \mathrm{H}_{48} \mathrm{O}_{24}$ & 575.1213 & 3.2 & $\begin{array}{c}\text { 693.1247; } 573.1050 ; 451.1032 ; \\
\text { 425.0874; } 411.0724 ; \text { 407.0772; } \\
\text { 289.0720; } 285.0406 ; 137.0245 ; \\
125.0245\end{array}$ & 2 \\
\hline 222 & A-Procyanidin trimer & 8.38 & $\mathrm{C}_{45} \mathrm{H}_{36} \mathrm{O}_{18}$ & 863.1840 & 1.3 & $\begin{array}{c}\text { 711.1358; } 693.1247 ; 573.1050 \\
\text { 451.1032; } 425.0874 ; \text { 411.0724; } \\
\text { 289.0720; } 285.0406 ; 137.0245 ; \\
125.0245\end{array}$ & 2 \\
\hline 223 & B-Procyanidin trimer & 8.71 & $\mathrm{C}_{45} \mathrm{H}_{38} \mathrm{O}_{18}$ & 865.2002 & 1.9 & $\begin{array}{c}\text { 713.1520; } 577.1334 ; 575.1216 ; \\
\text { 451.1032; } 425.0874 ; \text {;07.0772; } \\
\text { 289.0720; } 287.0562 ; 137.0244 ; \\
125.0244\end{array}$ & 2 \\
\hline 224 & B-Procyanidin pentamer & 8.92 & $\mathrm{C}_{75} \mathrm{H}_{62} \mathrm{O}_{30}$ & 720.1611 & 2.9 & $\begin{array}{c}\text { 863.1821; 693.1250; 577.1334; } \\
\text { 575.1216; 451.1032; 425.0874; } \\
\text { 289.0720; 287.0562; 137.0244; } \\
\text { 125.0244 }\end{array}$ & 2 \\
\hline 225 & A-Procyanidin tetramer & 9.60 & $\mathrm{C}_{60} \mathrm{H}_{48} \mathrm{O}_{24}$ & 575.1209 & 2.6 & $\begin{array}{l}\text { 863.1859; 711.1330; 693.1247; } \\
\text { 573.1050; 451.1032; 425.0874; } \\
\text { 411.0724; 407.0772; 289.0720; } \\
\text { 285.0406; 137.0245; } 125.0245\end{array}$ & 2 \\
\hline 226 & B-Procyanidin tetramer & 9.65 & $\mathrm{C}_{60} \mathrm{H}_{50} \mathrm{O}_{24}$ & 576.1288 & 2.5 & $\begin{array}{c}\text { 577.1334; } 575.1216 ; 451.1032 ; \\
\text { 425.0874; } 407.0772 ; 289.0720 ; \\
\text { 287.0562; } 245.0828 ; 137.0244 ; \\
125.0244\end{array}$ & 2 \\
\hline 227 & $\begin{array}{l}\text { A-Proanthocyanidin dimer } \\
\text { (galcat-cat) }\end{array}$ & 9.98 & $\mathrm{C}_{30} \mathrm{H}_{24} \mathrm{O}_{13}$ & 591.1151 & 1.2 & $\begin{array}{c}\text { 591.1118; } 465.0753 ; 439.0693 \\
\text { 303.0506; } 285.0406 ; 137.0245 ; \\
125.0245\end{array}$ & 2 \\
\hline 228 & B-Procyanidin pentamer & 10.15 & $\mathrm{C}_{75} \mathrm{H}_{62} \mathrm{O}_{30}$ & 720.1606 & 2.3 & $\begin{array}{l}\text { 863.1821; 737.1509; 693.1250; } \\
\text { 577.1334; 575.1216; 451.1032; } \\
\text { 425.0874; 407.0772; 289.0720; } \\
\text { 287.0562; 137.0244; } 125.0244\end{array}$ & 2 \\
\hline 229 & A-Procyanidin trimer & 10.54 & $\mathrm{C}_{45} \mathrm{H}_{36} \mathrm{O}_{18}$ & 863.1844 & 1.7 & $\begin{array}{c}\text { 711.1358; } 693.1247 ; 575.1198 ; \\
\text { 449.0878; } 423.0739 ; \text {;07.0772; } \\
\text { 289.0720; 285.0406; } 137.0245 ; \\
125.0245\end{array}$ & 2 \\
\hline
\end{tabular}


Table 4. Cont.

\begin{tabular}{|c|c|c|c|c|c|c|c|}
\hline ID & Name & Rt (min) & $\begin{array}{c}\text { Proposed } \\
\text { Formula }\end{array}$ & $\begin{array}{c}\text { Experimental } \\
\mathrm{m} / \mathrm{z}\end{array}$ & $\Delta(\mathrm{ppm})$ & Main Product Ions $(\mathrm{m} / \mathrm{z})$ & c. 1. \\
\hline 230 & B-Procyanidin pentamer & 10.61 & $\mathrm{C}_{75} \mathrm{H}_{62} \mathrm{O}_{30}$ & 720.1603 & 1.7 & $\begin{array}{c}\text { 721.1294; 577.1334; 575.1216; } \\
\text { 451.1032; 425.0874; 407.0772; } \\
\text { 289.0720; 287.0562; 137.0244; } \\
125.0244\end{array}$ & 2 \\
\hline 231 & B-Procyanidin tetramer & 10.75 & $\mathrm{C}_{60} \mathrm{H}_{50} \mathrm{O}_{24}$ & 576.1290 & 2.9 & $\begin{array}{c}\text { 577.1334; 575.1216; 451.1032; } \\
\text { 425.0874; 407.0772; 289.0720; } \\
\text { 287.0562; 245.0828; 137.0244; } \\
125.0244\end{array}$ & 2 \\
\hline 232 & A-Procyanidin pentamer & 10.82 & $\mathrm{C}_{75} \mathrm{H}_{60} \mathrm{O}_{30}$ & 719.1547 & 4.9 & $\begin{array}{c}\text { 1115.2210; 861.1715; 719.1268; } \\
\text { 577.1363; 451.1054; 449.0878; } \\
\text { 425.0880; 411.0740; 407.0772; } \\
\text { 289.0720; 285.0406; 137.0245; } \\
\text { 125.0245 }\end{array}$ & 2 \\
\hline 233 & A-Procyanidin tetramer & 10.94 & $\mathrm{C}_{60} \mathrm{H}_{48} \mathrm{O}_{24}$ & 575.1213 & 3.1 & $\begin{array}{c}575.1198 ; 449.0878 ; 423.0739 \\
407.0772 ; 289.0720 ; 285.0406 \\
137.0245 ; 125.0245\end{array}$ & 2 \\
\hline 234 & B-Procyanidin hexamer & 11.07 & $\mathrm{C}_{90} \mathrm{H}_{74} \mathrm{O}_{36}$ & 864.1883 & -2.8 & $\begin{array}{c}\text { 1151.2379; 865.1873; 863.1821; } \\
\text { 695.1427; 577.1334; 575.1216; } \\
\text { 451.1032; 425.0874; 407.0772; } \\
\text { 289.0720; 287.0562; 245.0828; } \\
\text { 137.0244; } 125.0244\end{array}$ & 2 \\
\hline 235 & B-Procyanidin hexamer & 11.42 & $\mathrm{C}_{90} \mathrm{H}_{74} \mathrm{O}_{36}$ & 864.1910 & 0.3 & $\begin{array}{c}\text { 1151.2379; 865.1873; 863.1821; } \\
\text { 695.1427; 577.1334; 575.1216; } \\
\text { 451.1032; 425.0874; 407.0772; } \\
\text { 289.0720; 287.0562; 245.0828; } \\
\text { 137.0244; } 125.0244\end{array}$ & 2 \\
\hline 236 & A-Procyanidin pentamer & 11.87 & $\mathrm{C}_{75} \mathrm{H}_{60} \mathrm{O}_{30}$ & 719.1527 & 2.1 & $\begin{array}{c}\text { 861.1613; 739.1692; 737.1482; } \\
\text { 689.2198; 577.1314; 573.1050; } \\
\text { 451.1032; 425.0874; 411.0724; } \\
\text { 407.0772; 289.0720; 285.0406; } \\
\text { 137.0245; } 125.0245\end{array}$ & 2 \\
\hline 237 & A-Procyanidin dimer & 12.02 & $\mathrm{C}_{30} \mathrm{H}_{24} \mathrm{O}_{12}$ & 575.1204 & 1.5 & $\begin{array}{c}\text { 449.0883; } 423.0725 ; 407.0762 \\
289.0720 ; 285.0406 ; 137.0245 \\
125.0245\end{array}$ & 2 \\
\hline 238 & A-Procyanidin hexamer & 12.02 & $\mathrm{C}_{90} \mathrm{H}_{72} \mathrm{O}_{36}$ & 863.1840 & 1.9 & $\begin{array}{l}575.1198 ; 539.0993 ; 449.0878 \\
423.0739 ; 407.0772 ; 289.0720 \\
285.0406 ; 137.0245 ; 125.0245\end{array}$ & 2 \\
\hline 239 & A-Procyanidin pentamer & 12.30 & $\mathrm{C}_{75} \mathrm{H}_{60} \mathrm{O}_{30}$ & 719.1530 & 2.5 & $\begin{array}{c}\text { 693.1247; 573.1050; 451.1032; } \\
\text { 425.0874; 411.0724; 407.0772; } \\
\text { 289.0720; 285.0406; 137.0245; } \\
125.0245\end{array}$ & 2 \\
\hline 240 & B-Procyanidin dimer & 12.35 & $\mathrm{C}_{30} \mathrm{H}_{26} \mathrm{O}_{12}$ & 577.1369 & 3.0 & $\begin{array}{l}\text { 577.1334; 451.1032; 425.0874; } \\
\text { 407.0772; 289.0720; 287.0568; } \\
\text { 245.0828; 137.02443; 125.0244 }\end{array}$ & 2 \\
\hline 241 & A-Procyanidin tetramer & 12.44 & $\mathrm{C}_{60} \mathrm{H}_{48} \mathrm{O}_{24}$ & 575.1212 & 2.9 & $\begin{array}{l}\text { 863.1859; 711.1358; 693.1247; } \\
\text { 575.1198; 449.0878; 423.0739; } \\
\text { 407.0772; 289.0720; 285.0406; } \\
\text { 137.0245; }\end{array}$ & 2 \\
\hline 242 & B-Procyanidin hexamer & 12.49 & $\mathrm{C}_{90} \mathrm{H}_{74} \mathrm{O}_{36}$ & 864.1878 & -2.8 & $\begin{array}{c}\text { 863.1859; 693.1280; 575.1198; } \\
\text { 539.0993; 449.0878; 423.0739; } \\
\text { 407.0772; 289.0720; 285.0406; } \\
\text { 137.0245; }\end{array}$ & 2 \\
\hline 243 & $\begin{array}{l}\text { A-Proanthocyanidin trimer } \\
\text { (cat-afz-galcat) }\end{array}$ & 12.64 & $\mathrm{C}_{45} \mathrm{H}_{36} \mathrm{O}_{18}$ & 863.1820 & -1.0 & $\begin{array}{c}\text { 711.1358; } 693.1247 ; 575.1198 \\
\text { 449.0878; } 433.0771 ; 407.0772 \\
\text { 301.0340; } 289.0720 ; 137.0245 \\
125.0245\end{array}$ & 2 \\
\hline 244 & A-Procyanidin tetramer & 13.15 & $\mathrm{C}_{60} \mathrm{H}_{48} \mathrm{O}_{24}$ & 575.1210 & 2.7 & $\begin{array}{l}\text { 573.1050; 451.1032; 425.0874; } \\
\text { 411.0724; 407.0772; 289.0720; } \\
\text { 285.0406; 137.0245; } 125.0245\end{array}$ & 2 \\
\hline 245 & B-Procyanidin trimer & 13.21 & $\mathrm{C}_{45} \mathrm{H}_{38} \mathrm{O}_{18}$ & 865.2013 & 3.4 & $\begin{array}{c}\text { 713.1520; } 577.1334 ; 575.1216 \\
\text { 451.1032; } 425.0874 ; 407.0772 \\
\text { 289.0720; } 287.0562 ; 137.0244 \\
125.0244\end{array}$ & 2 \\
\hline 246 & diA-Procyanidin trimer & 13.44 & $\mathrm{C}_{45} \mathrm{H}_{34} \mathrm{O}_{18}$ & 861.1694 & -2.3 & $\begin{array}{c}\text { 693.1247; 575.1198; 571.09454; } \\
\text { 449.0878; 289.0720; } 285.0406 \\
137.0245 ; 125.0245\end{array}$ & 2 \\
\hline
\end{tabular}


Table 4. Cont.

\begin{tabular}{|c|c|c|c|c|c|c|c|}
\hline ID & Name & Rt (min) & $\begin{array}{c}\text { Proposed } \\
\text { Formula }\end{array}$ & $\begin{array}{c}\text { Experimental } \\
\mathrm{m} / \mathrm{z}\end{array}$ & $\Delta(\mathrm{ppm})$ & Main Product Ions $(\mathrm{m} / \mathrm{z})$ & c. 1. \\
\hline 247 & A-Procyanidin pentamer & 14.11 & $\mathrm{C}_{75} \mathrm{H}_{60} \mathrm{O}_{30}$ & 719.1526 & 2.0 & $\begin{array}{l}\text { 863.1859; 711.1358; 693.1247; } \\
\text { 575.1198; 449.0878; 423.0739; } \\
\text { 407.0772; 289.0720; 285.0406; } \\
\text { 137.0245 }\end{array}$ & 2 \\
\hline 248 & A-Procyanidin hexamer & 14.80 & $\mathrm{C}_{90} \mathrm{H}_{72} \mathrm{O}_{36}$ & 863.1845 & 1.9 & $\begin{array}{c}\text { 863.1859; 693.1280; 575.1198; } \\
\text { 539.0993; 449.0878; 423.0739; } \\
\text { 407.0772; 289.0720; 285.0406; } \\
\text { 137.0245 }\end{array}$ & 2 \\
\hline 249 & B-Procyanidin tetramer & 15.14 & $\mathrm{C}_{60} \mathrm{H}_{50} \mathrm{O}_{24}$ & 576.1293 & 3.5 & $\begin{array}{c}\text { 577.1334; } 575.1216 ; 451.1032 ; \\
\text { 425.0874; } 407.0772 ; 289.0720 ; \\
\text { 287.0562; } 245.0828 ; 137.0244 ; \\
125.0244\end{array}$ & 2 \\
\hline
\end{tabular}

\section{Results and Discussion}

The identification of phenolic compounds is a critical issue in the phytochemical analysis research field. Phenolic compounds are, in fact, a structurally diverse class of compounds, encompassing a wide range of molecular weights, acid-base properties, and structure complexity [27]. Flavonoids, anthocyanins, and phenolic acids are often present as glycoconjugates to sugars or sugar derivatives and/or acylated to aliphatic and aromatic acids. Historically, the analysis of phenolic compounds has been based on UV-Vis spectroscopy due to the extensively aromatic systems in their structures [28]. However, in recent years, untargeted HRMS has become the foremost technique for phenolic compound identification as it allows extending the characterization to a wide range of compounds, also without the need for analytical standards [29]. Because of their extreme complexity, HRMS raw data cannot be handled without using software programs that render accessible the large datasets by $\mathrm{m} / \mathrm{z}$ extraction, adduct grouping, and feature alignment. Moreover, when highly composite phytocomplexes are analyzed by untargeted HRMS, there is a need for tools that simplify the datasets for a more accessible manual validation of the compounds.

For the characterization of $D$. alaternoides, untargeted HRMS followed by a suspect screening data processing was employed, based on a methodology that was previously implemented on Compound Discoverer 3.1 by our research group [24]. Because of the wide range of bond energies in phenolic compound structures (from the weak acetal to the strong aromatic bonds), the acquisition was performed with a three-stepped NCE of 20-50-80 and 20-40-60 for the positive and negative ion mode, respectively. To obtain a larger number of chromatographic points per peak, separate chromatographic runs for each polarity were preferred to polarity switching mode. Top 5 DDA mode is widely used for untargeted analysis with orbitrap-based instrumentation as it allows high-quality MS/MS spectra for the five most intense ions for each scan in full-scan mode [30]. Compound Discoverer is based on a system of blocks and nodes that can be customized by the user for the development of specific data-processing methods. For this purpose, an extensive database of 45,567 phenolic compound derivatives was generated by considering flavonoids, phenolic acids, and tannins in their free and conjugated to sugars and acids. The database, which was implemented in the mass list tool, was employed to filter the extracted and aligned features to remove calculated masses not included in the database. Manual validation was also aided by the compound class scoring tool, which matches the experimental MS/MS to theoretical fragmentation of the flavonoid or phenolic acid core. According to this approach, 16 and 233 phenolic compounds were identified and tentatively identified, respectively. The use of DDA mode for data acquisition allowed the annotation of several compounds even though some of them coeluted. In fact, as precursor ions are sequentially isolated and fragmented with an isolation window of $2 \mathrm{~m} / \mathrm{z}$, whenever compounds differing from more than $2 \mathrm{Da}$ coeluted, they could still be tentatively identified. In Figure 1, the classes of identified phenolics are reported in terms of the number of identifications and the total peak areas per 
class. The largest class of compounds was phenolic acids with 108 compounds, followed by flavonoids (87 compounds); proanthocyanidins ( 36 compounds); and, finally, anthocyanins (18 compounds). Despite being the less numerous, anthocyanins were the most abundant class in terms of total peak area with almost $45 \%$ of the total. Flavonoids and phenolic acids were equally distributed (with $28.2 \%$ and $25.5 \%$, respectively). Finally, proanthocyanidins comprised only the $1.7 \%$ of the total peak area. Identification for each class is discussed in the following sections.
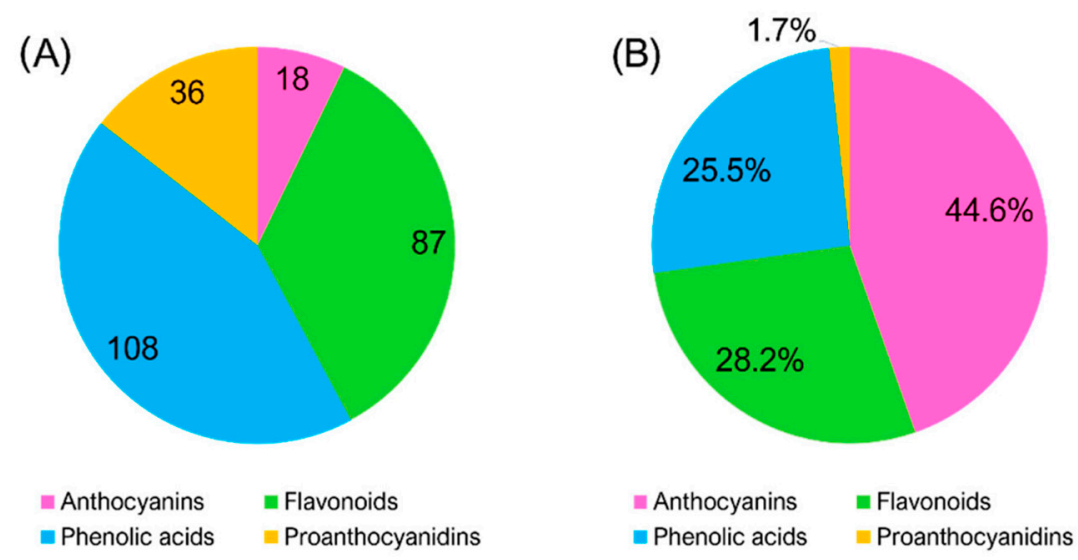

Figure 1. Pie chart reporting the tentatively identified classes of compounds in terms of (A) number of identifications and (B) total peak area.

\subsection{Anthocyanin and Flavonoid Composition}

Anthocyanins are a peculiar class of phenolic compounds synthesized via the phenylpropanoid pathways but differently from the other flavonoids, characterized by a positive charge on the oxygen of the C-ring of the basic flavonoid structure [31]. Because of this positive charge, anthocyanins are commonly determined in positive ion mode in the form of molecular ions $[\mathrm{M}]^{+}$since the corresponding adduct $[\mathrm{M}-2 \mathrm{H}]^{-}$in negative mode is generated with a noticeably lower sensitivity. In Table 1, the 18 tentatively identified anthocyanins are listed, while in Table S1 further details on the identification are reported (adducts, molecular weights, confirming peaks, and peak areas).

Despite their generally high peak areas (with compound 5 comprising alone almost $40 \%$ of the total peak area), the tentatively identified anthocyanins are numerically inferior compared to European and North American blueberries, for which more than 50 anthocyanins were previously reported [2]. Regarding the aglycones, methylated compounds were significantly under-expressed compared to V. myrtillus and V. corymbosum, with only two minor peonidin derivatives identified (compounds 12-13). Malvidin and petunidin derivatives, which are major constituents of blueberries, were not found. It is worth mentioning that malvidin derivatives have not been identified in $V$. floribundum, an Andean blueberry that grows in the same regions as D. alaternoides [19,20]. In a previous paper by Ma et al. [9], phenolic markers for discriminate North American and Neotropical blueberries were studied, comprising another member of the genus Disterigma (D. rimbachii). Malvidin derivatives were effectively under-expressed in Neotropical blueberries compared to North American ones. The absence of malvidin derivatives was also apparent from the color of the extract, which is significantly more reddish (and less purplish) than those of blueberry and bilberry.

Besides anthocyanins, 87 other flavonoids were tentatively identified in D. alaternoides, mostly flavanol derivatives. Among the several aglycones belonging to this class, quercetin derivatives were the most abundant with more than $97 \%$ of the total flavonol peak area, followed by minor amounts of kaempferol, myricetin, and isorhamnetin. The flavonol composition of Andean blueberry was noticeably similar to that of other genus Vaccinium species, except for laricitrin and syringetin derivatives, which were not identified in the D. alaternoides extract $[2,32]$. Similar to malvidin, laricitrin and syringetin are $O$-methylated 
compounds. Their simultaneous absence could indicate a lower degree of methylation in the flavonoid constituents of Andean blueberries compared to European and North American blueberries.

Flavonoids were analyzed in both positive and negative ion modes, with the latter providing generally higher ionization efficiencies. In Table 2, the annotated flavonoids were reported alongside some details, i.e., retention time, proposed formula, experimental $\mathrm{m} / \mathrm{z}$, accuracy, main diagnostic product ions, and confidence level in ESI(-), except for compounds 79,80 , and 88 , which were uniquely identified in ESI(+). In Table S2, further details were provided, including complete MS/MS spectra in both ion modes. The determination of the position of the glycoconjugation on the flavonol structure is a great analytical challenge when authentic standards are not available. The sugar-aglycone bond can undergo both heterolytic and homolytic cleavage in the negative ion mode, producing an aglycone ion $\left[\mathrm{Y}_{0}\right]^{-}$and a radical aglycone ion $\left[\mathrm{Y}_{0}-\mathrm{H}\right]^{-}$, respectively. Differently from the hydroxyl position on the aromatic rings (e.g., position 7 on the A-ring or position $4^{\prime}$ on the B-ring), when a sugar is bound to position 3 (on the non-aromatic C-ring of the flavanol structure), the homolytic cleavage is favored [33]. Based on these pieces of evidence, whenever the radical aglycone ion had a higher abundance than the aglycone ion, the compounds were ascribed to 3-O-monosaccharide derivatives. Whenever the aglycone ion was more abundant or in the case of more than one glycosylation, the position was not indicated, as positions 7 and $4^{\prime}$ are not distinguishable by HRMS [34]. In agreement with previous findings on other species of the Vaccinium genus, the majority of flavonols were 3-O-glycosylated [2].

\subsection{Phenolic Acid Composition}

To date, phenolic acids in berries from the Ericaceae family have been largely neglected to date compared to flavonoids and flavonoid derivatives, despite their interesting biological activities and high abundance in species of the Vaccinium genus [1]. Anthocyanin-rich matrices are often only analyzed in the positive ion mode [19], which is unsuitable for analyzing strong acid compounds. Moreover, whereas flavonoid structures are somehow consistent in different matrices, phenolic acids encompass a more comprehensive range of compounds, resulting in the need for several analytical standards for targeted analyses. In the case of $V$. floribundum, which is the most similar berry to $D$. alaternoides in terms of anthocyanin and flavonoid composition, no more than 7 phenolic acids have been reported so far by previous liquid chromatography coupled to MS analyses $[19,35,36]$. The reported phenolic acids comprised mainly hydroxycinnamic acids conjugated to quinic and shikimic acid, with the most abundant being chlorogenic acid. In the present paper, a total of 108 phenolic acids and phenolic acid derivatives have been tentatively identified in the Andean blueberry extract by HRMS analysis in the negative ion mode, a number that was significantly higher than reported for blueberries of the genus Vaccinium $[2,7,19,32,37]$. In Table 3 and Table S3, details of the annotated phenolic acids were reported.

Unlike previous studies on blueberries, the annotated phenolic acids presented a more significant structural variability and could be grouped into six main categories, i.e., arbutin conjugates, quinic and shikimic acid conjugates, hydroxycinnamic acid glycosides, hydroxybenzoic acid glucosides, coumaroyl iridoids, and free phenolic acids. The large number of tentative identifications, with tremendous structural heterogeneity and a high total peak area (more than 25\%), implied phenolic acids have a role more important than expected in the composition of $D$. alaternoides. Figure 2 shows the total peak area for each of the six classes of compounds. Arbutin derivatives were the most abundant compounds ( $40 \%$ of the total peak area), followed by quinic and shikimic acid conjugates (38\%). Hydroxycinnamic and hydroxybenzoic glycosides contributed to the total peak area with $10.6 \%$ and $7.2 \%$, while coumaroyl iridoids and free phenolic acids were present in minor amounts (ca. 2\%). 


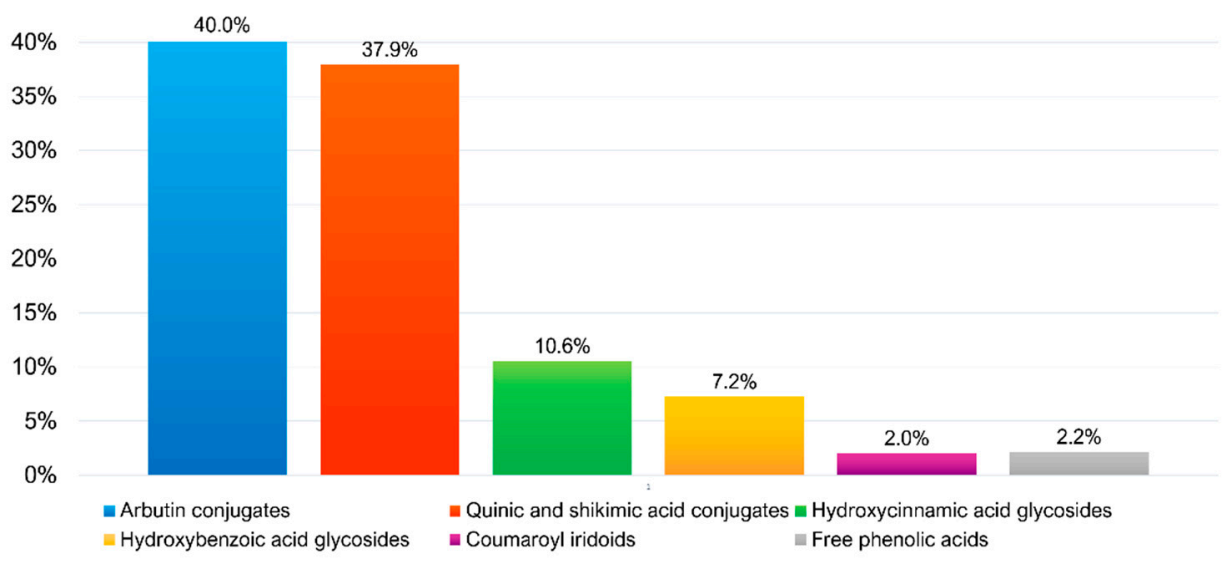

Figure 2. Bar chart reporting the total peak area per class of phenolic acids tentatively identified in Disterigma alaternoides extract.

Arbutin conjugates presented extremely high concentrations despite being numerically a minor class (10 out of 108 compounds). As a matter of fact, caffeoyl arbutin (compound 165) was the single most abundant phenolic acid in terms of peak area. Arbutin is a glucoside of hydroquinone primarily found in blueberry leaves [38] and is known for its skin-whitening properties [39] as well as its efficacy in the treatment of various urinary tract infections [40]. Caffeoyl arbutin was identified by a prior loss of $110 \mathrm{Da}$ (hydroquinone). This cleavage generates a dehydration on the sugar moiety due to the extremely strong $\mathrm{C}-\mathrm{O}$ phenolic bond of hydroquinone. Therefore, the subsequent sugar cleavage led to a loss of $144 \mathrm{Da}$ (glucose- $-2 \mathrm{H}_{2} \mathrm{O}$ ) rather than the usual neutral loss of $162 \mathrm{Da}$ (glucose$\mathrm{H}_{2} \mathrm{O}$ ). In a previous paper by Ieri et al. [41], several arbutin derivatives were identified in a bilberry leaf extract, including caffeoyl and coumaroyl arbutin, as well as their acetyl derivatives, in good agreement with our findings. Other than caffeoyl arbutin (compounds 163 and 165), other derivatives were identified, i.e., caffeoyl hexosyl arbutin (three isomers, compounds 153, 159, and 160), caffeoyl acetyl arbutin (two isomers, compounds 182 and 183), caffeoyl methoxyarbutin (compound 169), and hydroxybenzoyl arbutin (two isomers, compounds 161 and 180), which were identified with the same logic as described for caffeoyl arbutin. Considering how neglected phenolic acids generally are, arbutin derivatives are likely to be present in all blueberry fruits rather than be solely present in D. alaternoides.

The identified phenolic acid conjugates were mainly hydrophobic hydroxycinnamic derivatives (caffeoyl; coumaroyl; and, to a lesser extent, feruloyl, and sinapoyl conjugates) rather than hydrophile hydroxybenzoic derivatives. Gallic acid and its polymeric derivatives (gallotannins and ellagitannins) were scarcely represented, while several minor glycoconjugates of benzoic, hydroxybenzoic, and dihydroxybenzoic acid have been tentatively identified. Despite a large number of identified compounds (60 compounds), phenolic acid glycoconjugates represented just $18 \%$ of the total peak area, likely due to a large number of minor positional isomers. Among the other minor constituents, two coumaroyl iridoids (compounds 166-167) were tentatively identified; these compounds, which are characteristic of cranberry ( $V$. macrocarpon), are of great interest for their possible role in healing urinary tract infections [42].

\subsection{Proanthocyanidin Composition}

Proanthocyanidins are non-hydrolyzable oligomers of flavanols, mainly (epi)catechin and (epi)gallocatechin, and are distinguished into two subclasses according to their linkage. A-type proanthocyanidins present a double linkage from positions 7 and 8 on the ring A of the terminal unit to positions 2 and 4 on the ring $C$ of the extension unit $(2 \beta \rightarrow O \rightarrow 7 ; 4 \beta \rightarrow 8)$, while B-type proanthocyanidins present a single interflavanoid bond $(4 \beta \rightarrow 8)$. For simplicity, species with one or more A-type interflavanoid bonds are commonly defined as A-type proanthocyanidins [43]. The standard nomenclature is perfectly suitable for dimers, 
as there is either one A-type bond or one B-type bond. Nevertheless, it is worth specifying that, concerning oligomers with more than two units, there are more than two possibilities, e.g., in the case of trimers, two B-type bonds, two A-type bonds, and one for each kind, with the latter two both falling under the definition of A-type proanthocyanidins. For sake of clarity, whenever more than one A-type bond was present in the oligomer, a prefix was added to the name (compound 246). In Table 4 and Table S4, detailed data of the 36 tentatively identified proanthocyanidins were reported. Despite being efficiently ionized in both positive and negative ion mode, proanthocyanidins have been only analyzed in negative polarity for the generally higher ionization efficiency, the higher clarity of the MS/MS spectra, and the minor interference of contaminants and noise. Proanthocyanidin fragmentation pathways involve quinone methide (QM) fissions, retro-Diels-Alder (RDA) ring openings, and heterocyclic ring fissions (HRF). QM fissions generate two distinctive sections of the oligomer, named terminal and extension unit (or $\beta$ unit). While terminal unit ions deriving from $\mathrm{QM}$ fissions are independent of the linkage, i.e., $\left[\mathrm{M}_{\mathrm{T}}-\mathrm{H}\right]^{-}$, product ions of the extension unit are distinctive, i.e., $\left[\mathrm{M}_{\mathrm{E}}-3 \mathrm{H}\right]^{-}$and $\left[\mathrm{M}_{\mathrm{E}}-5 \mathrm{H}\right]^{-}$for $\mathrm{B}$ - and A-type oligomers, respectively. RDA C-ring opening generates neutral losses of the B-ring sections of the flavanol, producing diagnostic losses for the single flavanol (152.0423 and $168.0432 \mathrm{u}$ for catechin and gallocatechin, respectively). Finally, the HRF pathway generates confirming peaks deriving from the loss of the A-ring sections of the flavanol $(126.0317 \mathrm{u})$, independent from the flavanol's nature.

Compared to other proanthocyanidin-rich matrices, such as tea [44], strawberry [22], and even bilberry [2], the identified compounds were primarily A- and B-type procyanidins, oligomers of the sole catechin and epicatechin. A-type procyanidins were more abundant than B-type ones both in terms of the number of identifications (20 vs. 16) and the total peak area ( $74 \%$ vs. $26 \%)$, in good agreement with previously found for bilberry [2].

\section{Conclusions}

Wild neotropical berries are still an undisclosed rich source of bioactive compounds. In the present paper, almost 250 phenolic compound derivatives were tentatively identified, including anthocyanins, flavonoids, phenolic acids, and proanthocyanidins, in Disterigma alaternoides, an Andean blueberry of the unfamiliar genus Disterigma of the family Ericaceae. These results indicated, once more, the need for more profound and capillary knowledge on these exotic berry species. Many of the identified compounds are indeed known to exert important biological activities. The high number of tentative identifications was achieved thanks to a dedicated analytical platform based on HRMS and data analysis that allowed a comprehensive yet accessible phenol characterization. The annotated compounds were in general agreement with the composition of other blueberry, with high anthocyanin and flavanol glycoconjugated. The anthocyanin and flavonoid pattern, however, was more similar to that of other Andean blueberries, such as $V$. floribundum, with the absence of highly methylated malvidin, petunidin, and syringetin derivatives. Phenolic acids, which are a generally less investigated class of phenolic compounds, were instead the most numerous and heterogenous class. Several phenolic acids that conjugated to arbutin, which is present in the leaves of blueberry plants, were reported for the first time in blueberry fruit extracts. The extremely rich composition of $D$. alaternoides represents a pivotal result in the field of neotropical berries, which are emerging as possible "superfruits" for the biological activities of their compounds.

Supplementary Materials: The following are available online at https:/ /www.mdpi.com/article/10 .3390 / separations 8050058 / s1, Table S1: detailed identification data for the annotated anthocyanins in Disterigma alaternoides; Table S2: detailed identification data for the annotated flavonoids in Disterigma alaternoides; Table S3: detailed identification data for the annotated phenolic acids in Disterigma alaternoides; Table S4: detailed identification data for the annotated proanthocyanidins in Disterigma alaternoides. 
Author Contributions: Conceptualization, A.C.; methodology, S.E.A.; investigation, S.E.A., B.G.M. and C.M.M.; data curation, A.C.; writing—original draft preparation, A.C.; writing-review and editing, C.C. and S.P.; visualization, C.M.M.; supervision, A.L.C., resources: A.L.C. and A.L., project administration: A.L. All authors have read and agreed to the published version of the manuscript.

Funding: This research received no external funding.

Institutional Review Board Statement: Not applicable.

Informed Consent Statement: Not applicable.

Data Availability Statement: The data presented in this study are available in article.

Acknowledgments: The authors would like to thank Celia Vargas de la Cruz from the Academic Department of Pharmacology, Bromatology, and Toxicology at the National University of San Marcos in Lima, Perú for furnishing the certified D. alaternoides berries that were the object of the present study.

Conflicts of Interest: The authors declare no conflict of interest.

\section{References}

1. Eichholz, I.; Huyskens-Keil, S.; Rohn, S. Blueberry Phenolic Compounds: Fruit Maturation, Ripening and Post-Harvest Effects. In Processing and Impact on Active Components in Food; Elsevier Academic Press: Amsterdam, The Netherlands, 2015; ISBN 9780124047099.

2. Ancillotti, C.; Ciofi, L.; Rossini, D.; Chiuminatto, U.; Stahl-Zeng, J.; Orlandini, S.; Furlanetto, S.; Del Bubba, M. Liquid chromatographic/electrospray ionization quadrupole/time of flight tandem mass spectrometric study of polyphenolic composition of different Vaccinium berry species and their comparative evaluation. Anal. Bioanal. Chem. 2017, 409, 1347-1368. [CrossRef] [PubMed]

3. Guofang, X.; Xiaoyan, X.; Xiaoli, Z.; Yongling, L.; Zhibing, Z. Changes in phenolic profiles and antioxidant activity in rabbiteye blueberries during ripening. Int. J. Food Prop. 2019, 22, 320-329. [CrossRef]

4. Kim, J.G.; Kim, H.L.; Kim, S.J.; Park, K.-S. Fruit quality, anthocyanin and total phenolic contents, and antioxidant activities of 45 blueberry cultivars grown in Suwon, Korea. J. Zhejiang Univ. Sci. B 2013, 14, 793-799. [CrossRef]

5. Colak, N.; Primetta, A.K.; Riihinen, K.R.; Jaakola, L.; Grúz, J.; Strnad, M.; Torun, H.; Ayaz, F.A. Phenolic compounds and antioxidant capacity in different-colored and non-pigmented berries of bilberry (Vaccinium myrtillus L.). Food Biosci. 2017, 20, 67-78. [CrossRef]

6. Narwojsz, A.; Tańska, M.; Mazur, B.; Borowska, E.J. Fruit Physical Features, Phenolic Compounds Profile and Inhibition Activities of Cranberry Cultivars (Vaccinium macrocarpon) Compared to Wild-Grown Cranberry (Vaccinium oxycoccus). Plant Foods Hum. Nutr. 2019, 74, 300-306. [CrossRef]

7. Hokkanen, J.; Mattila, S.; Jaakola, L.; Pirttilä, A.M.; Tolonen, A. Identification of Phenolic Compounds from Lingonberry (Vaccinium vitis-idaea L.), Bilberry (Vaccinium myrtillus L.) and Hybrid Bilberry (Vaccinium x intermedium Ruthe L.) Leaves. J. Agric. Food Chem. 2009, 57, 9437-9447. [CrossRef] [PubMed]

8. Luteyn, J.L. Diversity, adaptation, and endemism in neotropical ericaceae: Biogeographical patterns in the vaccinieae. Bot. Rev. 2002. [CrossRef]

9. Ma, C.; Dastmalchi, K.; Flores, G.; Wu, S.-B.; Pedraza-Peñalosa, P.; Long, C.; Kennelly, E.J. Antioxidant and Metabolite Profiling of North American and Neotropical Blueberries Using LC-TOF-MS and Multivariate Analyses. J. Agric. Food Chem. 2013, 61, 3548-3559. [CrossRef] [PubMed]

10. Kalt, W.; Cassidy, A.; Howard, L.R.; Krikorian, R.; Stull, A.J.; Tremblay, F.; Zamora-Ros, R. Recent Research on the Health Benefits of Blueberries and Their Anthocyanins. Adv. Nutr. 2019, 11, 224-236. [CrossRef]

11. Yang, S.; Wang, C.; Li, X.; Wu, C.; Liu, C.; Xue, Z.; Kou, X. Investigation on the biological activity of anthocyanins and polyphenols in blueberry. J. Food Sci. 2021, 86, 614-627. [CrossRef] [PubMed]

12. Rutledge, G.A.; Sandhu, A.K.; Miller, M.G.; Edirisinghe, I.; Burton-Freeman, B.B.; Shukitt-Hale, B. Blueberry phenolics are associated with cognitive enhancement in supplemented healthy older adults. Food Funct. 2021, 12, 107-118. [CrossRef] [PubMed]

13. Stull, A. Blueberries' Impact on Insulin Resistance and Glucose Intolerance. Antioxidants 2016, 5, 44. [CrossRef] [PubMed]

14. Subbiah, V.; Zhong, B.; Nawaz, M.A.; Barrow, C.J.; Dunshea, F.R.; Suleria, H.A.R. Screening of Phenolic Compounds in Australian Grown Berries by LC-ESI-QTOF-MS/MS and Determination of Their Antioxidant Potential. Antioxidants 2020, 10, 26. [CrossRef] [PubMed]

15. Neamtu, A.-A.; Szoke-Kovacs, R.; Mihok, E.; Georgescu, C.; Turcus, V.; Olah, N.K.; Frum, A.; Tita, O.; Neamtu, C.; SzokeKovacs, Z.; et al. Bilberry (Vaccinium myrtillus L.) Extracts Comparative Analysis Regarding Their Phytonutrient Profiles, Antioxidant Capacity along with the In Vivo Rescue Effects Tested on a Drosophila melanogaster High-Sugar Diet Model. Antioxidants 2020, 9, 1067. [CrossRef]

16. De la Torre, L.; Navarrete, H.; Muriel, P.; Macía, M.J.; Balslev, H. Enciclopedia de las Plantas Útiles del Ecuador; Pontificia Universidad Católica del Ecuador: Quito, Ecuador, 2008; ISBN 9789978771358. 
17. Ortiz, J.; Marín-Arroyo, M.-R.; Noriega-Domínguez, M.-J.; Navarro, M.; Arozarena, I. Color, Phenolics, and Antioxidant Activity of Blackberry (Rubus glaucus Benth.), Blueberry (Vaccinium floribundum Kunth.), and Apple Wines from Ecuador. J. Food Sci. 2013, 78, C985-C993. [CrossRef]

18. Schreckinger, M.E.; Lotton, J.; Lila, M.A.; de Mejia, E.G. Berries from South America: A Comprehensive Review on Chemistry, Health Potential, and Commercialization. J. Med. Food 2010, 13, 233-246. [CrossRef] [PubMed]

19. Vasco, C.; Riihinen, K.; Ruales, J.; Kamal-Eldin, A. Chemical Composition and Phenolic Compound Profile of Mortiño (Vaccinium floribundum Kunth). J. Agric. Food Chem. 2009, 57, 8274-8281. [CrossRef]

20. Esquivel-Alvarado, D.; Muñoz-Arrieta, R.; Alfaro-Viquez, E.; Madrigal-Carballo, S.; Krueger, C.G.; Reed, J.D. Composition of Anthocyanins and Proanthocyanidins in Three Tropical Vaccinium Species from Costa Rica. J. Agric. Food Chem. 2020, 68, 2872-2879. [CrossRef] [PubMed]

21. Pedraza-Peñalosa, P. Systematics of the Neotropical Blueberry Genus Disterigma (Ericaceae). Syst. Bot. 2009, 34, 406-413. [CrossRef]

22. La Barbera, G.; Capriotti, A.L.; Cavaliere, C.; Piovesana, S.; Samperi, R.; Zenezini Chiozzi, R.; Laganà, A. Comprehensive polyphenol profiling of a strawberry extract (Fragaria $\times$ ananassa) by ultra-high-performance liquid chromatography coupled with high-resolution mass spectrometry. Anal. Bioanal. Chem. 2017, 409, 2127-2142. [CrossRef]

23. Cavaliere, C.; Antonelli, M.; Capriotti, A.L.; La Barbera, G.; Montone, C.M.; Piovesana, S.; Laganà, A. A Triple Quadrupole and a Hybrid Quadrupole Orbitrap Mass Spectrometer in Comparison for Polyphenol Quantitation. J. Agric. Food Chem. 2019, 67, 4885-4896. [CrossRef] [PubMed]

24. Cerrato, A.; Cannazza, G.; Capriotti, A.L.; Citti, C.; La Barbera, G.; Laganà, A.; Montone, C.M.; Piovesana, S.; Cavaliere, C. A new software-assisted analytical workflow based on high-resolution mass spectrometry for the systematic study of phenolic compounds in complex matrices. Talanta 2020, 209, 120573. [CrossRef] [PubMed]

25. Cacciola, N.A.; Cerrato, A.; Capriotti, A.L.; Cavaliere, C.; D'Apolito, M.; Montone, C.M.; Piovesana, S.; Squillaci, G.; Peluso, G.; Laganà, A.; et al. Untargeted Characterization of Chestnut (Castanea sativa Mill.) Shell Polyphenol Extract: A Valued Bioresource for Prostate Cancer Cell Growth Inhibition. Molecules 2020, 25, 2730. [CrossRef]

26. Schymanski, E.L.; Jeon, J.; Gulde, R.; Fenner, K.; Ruff, M.; Singer, H.P.; Hollender, J. Identifying Small Molecules via High Resolution Mass Spectrometry: Communicating Confidence. Environ. Sci. Technol. 2014, 48, 2097-2098. [CrossRef] [PubMed]

27. Tsao, R. Chemistry and Biochemistry of Dietary Polyphenols. Nutrients 2010, 2, 1231-1246. [CrossRef] [PubMed]

28. Baeza, G.; Sarriá, B.; Bravo, L.; Mateos, R. Exhaustive Qualitative LC-DAD-MS n Analysis of Arabica Green Coffee Beans: Cinnamoyl-glycosides and Cinnamoylshikimic Acids as New Polyphenols in Green Coffee. J. Agric. Food Chem. 2016, 64, 9663-9674. [CrossRef]

29. Piovesana, S.; Cavaliere, C.; Cerrato, A.; Montone, C.M.; Laganà, A.; Capriotti, A.L. Developments and pitfalls in the characterization of phenolic compounds in food: From targeted analysis to metabolomics-based approaches. TrAC Trends Anal. Chem. 2020, 133, 116083. [CrossRef]

30. Barbier Saint Hilaire, P.; Rousseau, K.; Seyer, A.; Dechaumet, S.; Damont, A.; Junot, C.; Fenaille, F. Comparative Evaluation of Data Dependent and Data Independent Acquisition Workflows Implemented on an Orbitrap Fusion for Untargeted Metabolomics. Metabolites 2020, 10, 158. [CrossRef]

31. Khoo, H.E.; Azlan, A.; Tang, S.T.; Lim, S.M. Anthocyanidins and anthocyanins: Colored pigments as food, pharmaceutical ingredients, and the potential health benefits. Food Nutr. Res. 2017, 61, 1361779. [CrossRef]

32. Liu, S.; Marsol-Vall, A.; Laaksonen, O.; Kortesniemi, M.; Yang, B. Characterization and Quantification of Nonanthocyanin Phenolic Compounds in White and Blue Bilberry (Vaccinium myrtillus) Juices and Wines Using UHPLC-DAD-ESI-QTOF-MS and UHPLC-DAD. J. Agric. Food Chem. 2020, 68, 7734-7744. [CrossRef]

33. Lu, L.; Song, F.-R.; Tsao, R.; Jin, Y.-R.; Liu, Z.-Q.; Liu, S.-Y. Studies on the homolytic and heterolytic cleavage of kaempferol and kaempferide glycosides using electrospray ionization tandem mass spectrometry. Rapid Commun. Mass Spectrom. 2010, 24, 169-172. [CrossRef] [PubMed]

34. Pikulski, M.; Brodbelt, J.S. Differentiation of flavonoid glycoside isomers by using metal complexation and electrospray ionization mass spectrometry. J. Am. Soc. Mass Spectrom. 2003, 14, 1437-1453. [CrossRef] [PubMed]

35. Baenas, N.; Ruales, J.; Moreno, D.A.; Barrio, D.A.; Stinco, C.M.; Martínez-Cifuentes, G.; Meléndez-Martínez, A.J.; García-Ruiz, A. Characterization of andean blueberry in bioactive compounds, evaluation of biological properties, and in vitro Bioaccessibility. Foods 2020, 9, 1483. [CrossRef] [PubMed]

36. Prencipe, F.P.; Bruni, R.; Guerrini, A.; Rossi, D.; Benvenuti, S.; Pellati, F. Metabolite profiling of polyphenols in Vaccinium berries and determination of their chemopreventive properties. J. Pharm. Biomed. Anal. 2014, 89, 257-267. [CrossRef]

37. Bujor, O.-C.; Le Bourvellec, C.; Volf, I.; Popa, V.I.; Dufour, C. Seasonal variations of the phenolic constituents in bilberry (Vaccinium myrtillus L.) leaves, stems and fruits, and their antioxidant activity. Food Chem. 2016, 213, 58-68. [CrossRef]

38. Ștefănescu, B.E.; Szabo, K.; Mocan, A.; Crişan, G. Phenolic Compounds from Five Ericaceae Species Leaves and Their Related Bioavailability and Health Benefits. Molecules 2019, 24, 2046. [CrossRef] [PubMed]

39. Zhu, W.; Gao, J. The Use of Botanical Extracts as Topical Skin-Lightening Agents for the Improvement of Skin Pigmentation Disorders. J. Investig. Dermatol. Symp. Proc. 2008, 13, 20-24. [CrossRef] [PubMed]

40. Geetha, R.V.; Roy, A.; Lakshmi, T. Nature's weapon against urinary tract infections. Int. J. Drug Dev. Res. 2011, 3, 85-100. 
41. Ieri, F.; Martini, S.; Innocenti, M.; Mulinacci, N. Phenolic Distribution in Liquid Preparations of Vaccinium myrtillus L. and Vaccinium vitis idaea L. Phytochem. Anal. 2013, 24, 467-475. [CrossRef] [PubMed]

42. Turner, A.; Chen, S.-N.; Nikolic, D.; van Breemen, R.; Farnsworth, N.R.; Pauli, G.F. Coumaroyl Iridoids and a Depside from Cranberry (Vaccinium macrocarpon). J. Nat. Prod. 2007, 70, 253-258. [CrossRef]

43. Howell, A.B.; Reed, J.D.; Krueger, C.G.; Winterbottom, R.; Cunningham, D.G.; Leahy, M. A-type cranberry proanthocyanidins and uropathogenic bacterial anti-adhesion activity. Phytochemistry 2005, 66, 2281-2291. [CrossRef] [PubMed]

44. Dou, J.; Lee, V.S.Y.; Tzen, J.T.C.; Lee, M.-R. Identification and Comparison of Phenolic Compounds in the Preparation of Oolong Tea Manufactured by Semifermentation and Drying Processes. J. Agric. Food Chem. 2007, 55, 7462-7468. [CrossRef] [PubMed] 